Treatment of Comorbid Conduct Problems and Depression in Youth: A Pilot Study

\author{
Jennifer C. Wolff
}

\begin{abstract}
Dissertation Submitted to the Faculty of Virginia Polytechnic Institute and State University In partial fulfillment of the requirements for the degree of Doctor of Philosophy In Psychology
\end{abstract}

Committee Members:

Thomas H. Ollendick, Ph.D., Committee Chair

Lee D. Cooper, Ph.D., Committee Member

Julie A. Dunsmore, Ph.D., Committee Member

Angela S. Scarpa, Ph.D., Committee Member

February 19, 2008

Blacksburg, VA

Keywords: Conduct problems, depression, treatment, adolescent 
Treatment of Comorbid Conduct Problems and Depression in Youth: A Pilot Study

Jennifer C. Wolff

\begin{abstract}
The purpose of this study was to pilot a cognitive behavioral treatment protocol for adolescents with co-occurring conduct problems and depression and to examine changes in diagnostic status, symptomatic improvement, and related areas of functioning associated with this treatment. Manualized treatment consisted of 11 to 16 sessions that targeted problem solving skills, cognitive restructuring, affect regulation skills, and parenting strategies. A non-concurrent multiple baseline design was used to assess the effectiveness of the intervention. A sample of five adolescents, aged 11 to 14 years, participated; all five families completed the protocol. Measures of diagnostic status, symptoms, and global impairment were administered at baseline, post-treatment, and 1-month post-treatment. Decreases in depression and conduct problem symptoms as well as changes in diagnostic status were evident for three of the five participants. Clinician severity ratings improved for all of the children at post-treatment. Preliminary results suggest that integrated cognitive behavioral treatment for adolescents with conduct problems and depression is associated with symptomatic improvement as well. Results of this study have implications for improving treatment outcomes for youth with these comorbid conditions and may also lead to increased conceptual understanding of the nature of these comorbid problems. Randomized clinical trials are required to assess the effectiveness of this combined treatment program.
\end{abstract}


Table of Contents

Abstract

Table of Contents

List of Tables/Figures

iv

Introduction

Method

16

Participants

17

Measures

19

Procedures

26

Results

30

Discussion

45

References

56

Tables

68

Figures

80

Appendix A

94

Curriculum Vita

100 


\section{List of Tables/Figures}

Table 1: Participant Characteristics

Table 2: Mother and Child Weekly Symptom Ratings Based on the Conduct Problem and

Depression Rating Scale

Table 3: Participant Diagnoses by Phase of Treatment 70

Table 4: Behavior Checklist T-Scores for Each Participant and Overall Means

Table 5: Disruptive Behavior Disorder Rating Scale and Childhood Depression Inventory Scores

Table 6: Family Environment and Emotion Regulation Ratings 76

Table 7: Ratings of Mother-Child Problem Solving Task 78

Table 8: Mother and Child Ratings of Improvement 79

Figure 1: Mother and Child Weekly Ratings of ODD Symptoms 80

Figure 2: Mother and Child Weekly Ratings of Depression Symptoms

Figure 3: Clinician Ratings of Severity of Illness 86

Figure 4: Clinician Ratings of Global Improvement 
Treatment of Comorbid Conduct Problems and Depression in Youth

Introduction

Despite their divergent presentations at the symptom level, conduct problems and depression are highly comorbid conditions in childhood and adolescence (Angold, Costello, \& Erkanli, 1999; Wolff \& Ollendick, 2006). According to the Diagnostic and Statistical Manual of Mental Disorders (DSM-IV; American Psychiatric Association, 2000), symptoms associated with oppositional defiant disorder (ODD) include argumentativeness, loss of temper, and disregard for authority. Symptoms of conduct disorder (CD) comprise more severe forms of aggression including physical fighting, property destruction, and other delinquent acts. Symptoms of depression, on the other hand, relate more to internalized feelings of sadness and loneliness as well as loss of energy, apathy, and sleep problems. Although symptomatic disparities exist, these disorders occur at rates significantly higher than would be expected by chance alone (Angold et al., 1999).

Moreover, the overlap of these disorders is associated with a number of negative outcomes, the most severe being increased risk of suicidal behavior. Youth diagnosed with both these disorders are two times more likely to attempt suicide than those with depression alone and eight times more likely to make an attempt than those with conduct problems alone (Lewinsohn, Rohde, \& Seeley, 1995, 1995). In addition, comparisons of those with pure and comorbid conduct problems and depression have shown that those with the comorbid presentation have more emotional symptoms, substance abuse, and greater functional impairment (Ezpeleta, Domènech, \& Angold, 2006; Fleming, Boyle, \& Offord, 1993; Harrington, Fudge, Rutter, Pickles, \& Hill, 1991). 
Given the high rates of overlap between conduct problems and depression and their associations with negative outcomes, interventions geared at treating these co-occurring conditions in youth are needed. While much is known about effectively treating these disorders alone, no treatment to date has effectively treated these disorders in combination. To address this problem, an integrated approach to treatment is proposed that simultaneously addresses deficiencies and trains skills relevant to the treatment of both disorders. Such a combined approach would allow ongoing attention to both problems and a merging of treatment principles and techniques that specifically target conduct problems and depression. Accordingly, the current study aimed to develop and test an integrated treatment for conduct problems with the goal of improving treatment outcomes for youth with this comorbid presentation.

Prevalence. Studies of comorbidity between depression and conduct problems suggest that each of these disorders is associated with increased risk for the other. In a meta-analysis completed on child and adolescent community samples, 1.8-14.7\% met criteria for ODD or CD and $0.3-18.0 \%$ met criteria for depression (Angold et al.,1999). In reference to comorbidity, however, 4.4-83.3\% of those with depression also met criteria for ODD or CD while 2.2-45.9\% of those with ODD or CD also met criteria for depression. The median odds ratio between CD and depression was 6.6 (95\% CI: 4.4 to 11.0$)$. Thus, those who have one disorder appear to be at increased risk for the other.

Among studies of clinic-referred youth, rates of comorbidity between these disorders are even higher. In a study conducted by Greene et al. (2002), more than $30 \%$ of clinically referred children diagnosed with severe major depression also met criteria for conduct problems. Conversely, of those with conduct problems, more than $50 \%$ met criteria for major depression. In examining rates of comorbidity as they relate to the specific disorders comprising conduct 
problems, comorbid depression appears to be more prevalent in those with $\mathrm{CD}$ compared to those with ODD. For example, in a study of inpatient adolescents, Arredondo and Butler (1994) found that $27 \%$ of the adolescents with ODD (compared to $76 \%$ with CD) met the diagnosis of a mood disorder. The frequent co-occurrence of these problems highlights the need for effective interventions designed to address multiple problems

Age of Onset. Although researchers differ in opinion on the sequence of onset between conduct problems and depression, both $\mathrm{CD}$ and depression tend to increase in prevalence during adolescence (Cohen et al., 1993). Some researchers suggest that conduct problems emerge first and the ensuing social failures experienced by these children and adolescents contribute to the development of depression (see Capaldi, 1992). Others, however, suggest that depression may emerge first followed by the development of conduct problems as a means of acting out or compensating for some of the internalized feelings associated with depression.

Many clinical and community based studies have found that conduct problems generally precede rather than follow the development of depression. For example, Biederman, Faraone, Mick, and Lelon (1995) reported that the age at onset for ODD and CD was six to seven years of age, compared to about eight years of age for depression. Among hospitalized girls in another study, the first symptom of CD was reported to occur at 8.2 years of age and that of depression at 13.5 years of age (Zoccolillo \& Rogers, 1991). In contrast, Kovacs, Paulauskas, Gatsonis, and Richards (1988) found that depression was more likely to precede conduct problems. In their study of 8- to 13-year-old children, more depressed children with comorbid CD developed depression prior to $\mathrm{CD}(56 \%)$ rather than $\mathrm{CD}$ prior to depression $(25 \%)$. More recently, it was found that in a sample of 382 children conduct problems preceded depression for $70.4 \%$ of participants (Ezpeleta et al., 2006). 
Thus, it appears that conduct problems may precede depression in most cases; however, in a significant minority of cases, depression precedes conduct problems. Overall, studies suggest that the prevalence of comorbidity reaches a peak around middle adolescence (Beyers \& Loeber, 2003; Loeber, Russo, Stouthamer-Loeber, \& Lahey, 1994). This phenomenon may be due to the fact that conduct problems tend to decrease as children move out of adolescence and into adulthood while depression tends to increase as children move into adolescence and then into adulthood.

Gender. Several studies have found gender differences in rates of comorbid conduct problems and depression. In terms of individual disorders, the rate of CD is considerably higher for adolescent males than for adolescent females (Robins \& Price, 1991), whereas the prevalence of depression is higher for adolescent females (Lewinsohn, Hops, Roberts, Seeley, \& Andrews, 1993). In a review of child and adolescent population studies, Zoccolillo (1992) found that the co-occurrence of these disorders is most likely in boys in preadolescence, diminishing in late adolescence and into adulthood, but most likely in girls during mid-adolescence and into adulthood. Overall, such studies have demonstrated that co-occurring conduct problems are more likely to occur in males than in females although some have found varying rates depending on parent or teacher reports (Keiley, Lofthouse, Bates, Dodge, \& Pettit, 2003). However, the severity of conduct problems in girls is greater than that for boys when comorbid disorders are taken into account (Loeber \& Keenan, 1994).

Paths to comorbidity. Researchers have hypothesized different causal relationships between depression and conduct problems (see Wolff \& Ollendick, 2006, for review). Experiencing early depression may impede social development, creating dyadic conflict and amplifying risk for negative reactions from others that lead to later conduct problems (Kovacs, 
Paulauskas, Gatsonis, \& Richards, 1988; Rudolph, Mannen, \& Burge, 1994). In contrast, conduct problems may emerge first and the disruption in interpersonal functioning may, in turn, promote depressive symptomology. Capaldi $(1991,1992)$ demonstrated support for this second hypothesis, in what she terms a dual-failure hypothesis. The model posits that conduct problems interfere with normal socialization and development as they lead to a chain reaction of developmental failures. The combination of lack of competence and negative reactions from others results in pervasive failures in adaptation and subsequently contributes to a child's vulnerability to the onset of depressive symptoms (Capaldi \& Stoolmiller, 1999). For example, higher levels of conduct problems are associated with peer rejection, problems in the parent-child relationship, and failure to develop academic skills (e.g., Capaldi, 1991). Such consequences often affect social development and contribute to associations with deviant peers, negative attitudes, and may in turn contribute to depressed mood. The dual failure pathway was supported in longitudinal analyses involving high risk boys (Capaldi, 1992; Capaldi \& Stoolmiller, 1999).

In contrast, other studies have proposed a dynamic link between conduct problems and depression that occurs concurrently and prospectively (Lahey, Loeber, Burke, Rathouz, \& McBurnett, 2002; Fergusson et al., 1996). It seems likely that a transactional process by which the child's behavior interacts with the environment over time to create new risk factors or problem behaviors is another path to co-occurring conduct problems and depression. The relationship may also be asymmetrical, with early conduct problems having a greater effect on depression than the reverse (Loeber \& Keenan, 1994).

Implications of Comorbid Depression and Conduct Problems. The importance of treating comorbid depression and conduct problems is heightened by the elevated risk for negative outcomes in adolescents with both disorders (Nottelmann \& Jensen, 1997). A recent study 
examined differences between those with pure and comorbid conduct problems and depression in a clinic referred sample (Ezpeleta et al., 2006). Comorbid children differed from the pure conduct group in that the former were more frequently angry and resentful, used weapons less frequently, set more fires, were less distracted, had more severe somatic complaints and anxiety, and were more globally functionally impaired. On the other hand, comorbid children, in comparison with the pure depressive group, had fewer sleep problems, more psychomotor symptoms (girls), fewer difficulties with appetite (boys), more somatic complaints, higher severity of anxiety symptoms and more functional impairment at school, home and in their interpersonal relationships. Overall, the co-occurrence of conduct and depressive disorders exacerbated emotional symptoms and functional impairment. Similarly, other studies have shown that in comparison with pure depression, comorbid children have been found to manifest more irritability (Harrington, Fudge, Rutter, Pickles, \& Hill, 1991), more substance abuse (Fleming, Boyle, \& Offord, 1993), and more academic problems and poorer global functioning (Lewinsohn et al., 1995). The comorbid group, in comparison with pure conduct problems, has been found to have a higher prevalence of destruction of property, running away, and substance abuse (Fleming et al., 1993) but less stealing, destructiveness, fighting/bullying and violent assaults (Simic \& Fombonne, 2001).

Moreover, youth with comorbid conduct problems and depression have a higher prevalence of suicidal ideation and suicide attempts (Capaldi, 1991, 1992; Fleming et al., 1993; Lewinsohn et al., 1995). In fact, one study showed that $38.9 \%$ of youth with comorbid depression and conduct problems had a history of one or more suicide attempts compared to $19 \%$ of those with major depression alone and $4.7 \%$ of those with pure conduct problems (Lewinsohn et al., 1995). Despite these implications, few studies have examined the treatment of youth with 
comorbid conduct problems and depression (see Rapp \& Wodarski, 1997; Rohde, Clarke, Mace, Jorgensen, \& Seeley, 2004).

Longitudinally, youths with comorbid conduct problems and depression are also prone to increased long-term problems in functioning (including adult criminality), compared with depressed-only youth (Capaldi, 1992; Harrington, Fudge, Rutter, \& Pickles, 1991; Kovacs, Paulauskas, Gatsonis, \& Richards, 1988). In a long term follow-up of depressed youth with and without comorbid conduct problems, the comorbid group evidenced high service utilization rates and costs in adulthood. More specifically, inpatient care and criminal justice services were used more frequently by the comorbid group and total costs were significantly higher. Thus, the high and enduring costs of comorbidity give further reason for the development of effective treatments.

Review of Treatments for Depression

Psychosocial treatments for youth with depression have advanced considerably over the past two decades. Cognitive behavioral therapy (CBT) has consistently received strong empirical support, as has interpersonal psychotherapy, for depressed youth (Curry, 2001; Curry, Wells, Lochman, Craighead, \& Nagy, 2003; Kaslow \& Thompson, 1998). Treatments along these lines have met the criteria for either a well-established treatment or probably efficacious treatment for youth with depression (Chorpita et al., 2002; Kaslow \& Thomson, 1998; Seligman, Goza, \& Ollendick, 2004). Effect sizes for CBT compared to a waitlist control, placebo, or alternative treatment range from -.01 to .94 at post-treatment (Compton et al., 2004; Treatment for Adolescents with Depression Study (TADS) Team, 2004).

CBT treatments for youth depression place varying emphasis on the cognitive and behavioral components of care. According to Beck's cognitive triad (1995), depressed persons 
maintain a negative schema of the self and seek out information concordant with that distorted point of view in their surroundings and interactions (1995). Specifically, these individuals view the self as inadequate, the world as unfair, and the future as hopeless. This "depressogenic" outlook, in turn, serves to filter out information inconsistent with this schema. The behavioral component of treatments for depression emphasizes various skill deficits. In particular, skill deficits in the domains of coping skills, interpersonal relationships, social problem solving, and participation in pleasant activities are emphasized (Kazdin \& Weisz, 1998). In all, CBT for depressed youth views these individuals as subject to schemas and cognitive distortions that cast experiences in an overly negative manner and as lacking important skills needed to create and maintain supportive relationships and to regulate emotion through daily activity.

With support from the National Institute of Mental Health, the Treatment for Adolescents with Depression Study (TADS; 2004) recently investigated the effectiveness of four treatments for adolescents with major depressive disorder: fluoxetine, CBT, combined treatment, and pill placebo. The CBT component of treatment was designed to meet best practice standards as it compiled components of various empirically supported treatments for youth depression into one manualized treatment protocol. A total of 439 youth, aged 12 to 17 years were treated as part of this multi-site, randomized control trial. It should be noted that those with severe CD were excluded from the study, but $13.2 \%$ met criteria for ODD.

Results of the study suggest that combined CBT and medication treatment are the most efficacious treatment for adolescent depression. Surprisingly, however, comparisons of the CBT treatment to the placebo control condition were not significantly different. Furthermore, CBT alone received a relatively low response rate of $43 \%$. These findings are in contrast to those obtained in earlier trials in which up to $70 \%$ of youth responded (Kazdin \& Weisz, 1998). It was 
suggested that this lower rate of response might be related to the sample composition since patients in this trial had more severe and chronic depression and higher rates of comorbidity than participants in previous CBT trials. As a result, youth in this trial may have fared more poorly with treatment (TADS, 2004).

\section{Review of Treatments for Conduct Problems}

For approximately the past 40 years, parent management training (PMT) programs have been the single most successful treatment approach for reducing conduct problems (Brestan \& Eyberg, 1998). Many treatment variants of PMT have been disseminated and vary widely in their relative emphasis on social learning theory, operant conditioning, parent-child relationship enhancement, and cognitive skills-training. In general, PMT teaches parents to use contingency management procedures to improve coercive interactions between parents and their children. In addition, PMT programs focus on 1) improving the quality of parent-child interactions (e.g., use of special time); 2) changing antecedents to behaviors to increase the likelihood of prosocial behaviors in the future (contingent reinforcement); 3) improving parents' abilities to monitor and supervise their children; and 4) teaching parents more effective discipline strategies (e.g., using differential attention, rewarding positive behaviors, and time out from reinforcement (Barkley, 1997; Frick, 2001; McMahon \& Forehand, 2003). Deficits in these specific aspects of parenting have been consistently linked to child oppositional behavior in a large body of research (Frick, 1998, 2001; Kazdin, 1995, 2005; Patterson, Reid, \& Dishion, 1992). Indeed, an impressive body of research has documented the efficacy of PMT, and these findings are sufficiently compelling to qualify PMT as an empirically validated, well-established treatment (see Brestan \& Eyberg, 1998, for review). 
An alternative approach to the treatment of youth with conduct problem behaviors is based on the finding that these youth show distortions and deficiencies in social information processing and problem solving (see Crick \& Dodge, 1994). CBT treatments for conduct problems focus on improving anger control, social skills, and problem-solving skills (e.g., Kazdin, Siegel, \& Bass, 1992; Lochman \& Wells, 1996; Spivack \& Shure, 1982; WebsterStratton, Reid, \& Hammond, 2001). In general, these programs focus on the thought processes involved in evaluating interpersonal situations and teaching youth a step-by-step process for solving interpersonal problems. Some evidence suggests that older children (11-13 years old) profit more from this treatment than younger children (5-7 years old; Durlak, Fuhrman, \& Lampman, 1991).

In a review of 82 treatment studies for youth with conduct problems, two variants of parent training met the stringent criteria for well-established interventions and several CBT programs were identified as "probably efficacious" (Brestan \& Eyberg, 1998). In a metaanalysis, Serketich and Dumas (1996) reported a mean effect size for parent training $(d=.86)$ that was notably higher than the mean effect size for CBT ( $d=.23$; Bennett \& Gibbons, 2000). However, a more recent meta-analysis found more comparable results with an effect size of 0.47 for parent training and 0.35 for child focused CBT (McCart, Priester, Davies, \& Azen, 2006).

\section{Efficacy of Treatments in Comorbid Populations}

While research has provided us with relatively effective ways of independently treating conduct problems or depression, much less is known about effective means of treating these disorders in combination. Early medication trials suggested that those with co-occurring disorders were less responsive to interventions than those with a single disorder. For example, one study found that depressed adolescents with comorbid conduct problems were less 
responsive to medication than those having co-occurring disorders (Hughes et al., 1990). In another medication trial, Puig-Antich (1982) reported that imipramine reduced depressive and conduct behaviors in preadolescents. However, these findings have not been replicated and may not apply to psychosocial interventions.

More recent studies have shown that the presence of comorbidity may not predict or moderate responses to psychosocial treatments. Costin and Chambers (2007), for example, divided study participants into three groups: (1) those with ODD alone, (2) those with ODD plus ADHD, and (3) those with ODD, ADHD, and an emotional disorder (either anxiety or depression). Contrary to the study's hypotheses, comorbidity did not predict poorer treatment outcome in response to parent training. In fact, all three groups showed improvements in oppositionality and, in fact, when assessed at post-treatment and 5-month follow-up the rates of improvement were greater for those in the comorbid groups compared to those with ODD alone. Moreover, these results held true when a measure of clinically significant change was applied. Thus, symptoms targeted by the intervention improved despite the presence of comorbidity.

Another study targeted oppositionality and examined the moderating role of depression symptoms (defined by CBCL scores) on treatment outcomes for those who received various combinations of parent, child, and teacher focused interventions (Beauchaine, Webster-Stratton, \& Reid, 2005). Analyses of moderation showed that interventions that included parent training were the most effective for children who scored below the median on the CBCL Anxious/Depressed subscale, whereas all intervention combinations were equally effective for children with elevated Anxious/Depressed scores. While results of this study revealed that cooccurring symptoms of Anxiety/Depression moderated treatment response, they did not predict negative outcomes in oppositional youth. 
However, other studies involving youth with comorbid conduct problems and depression suggest that treatment outcomes are specific to the targeted disorder and may not improve cooccurring symptoms. Such findings are consistent with the general psychotherapy literature in which outcome measures that specifically match targeted problems yield a larger average effect size (ES) than equally specific but unmatched outcome measures ( $E S=0.60$ vs. 0.30; see Weisz, Weiss, Han, Granger, \& Morton, 1995). In other words, the effects of treatment tend to be specific to the domains targeted in treatment.

Along these lines, a randomized clinical trial for adolescent depression demonstrated that CBT exerted specific effects on depression related variables, but there were no significant treatment effects on conduct disorder symptoms (Kolko, Brent, Baugher, Bridge, \& Birmaher, 2000). Similarly, Rohde and colleagues (2004) examined the efficacy of the Adolescent Coping with Depression Course (CWD-A), a cognitive behavioral group intervention for adolescent depression, in comparison to a life skills (LS) control treatment. Adolescents with comorbid CD and depression were referred from a juvenile justice setting and randomly assigned to treatment conditions. Given that the CWD-A intervention contained components of cognitive-behavioral interventions previously shown to be efficacious in the treatment of conduct problems (e.g., problem solving; Kazdin \& Weisz, 1998), it was hypothesized that this intervention would affect symptoms of both depression and conduct problems. However, it should be noted that although the CWD-A treatment contained a problem solving component, it was not designed for the treatment of CD per se nor were conduct problem behaviors a primary target of the intervention. At the end of treatment, although recovery rates for major depressive disorder were significantly greater in CWD-A compared to the life skills control treatment, $90 \%$ of the sample retained their $\mathrm{CD}$ diagnosis. Moreover, differences in $\mathrm{CD}$ diagnoses between the two groups were not 
significantly different at post-treatment and follow-up. Thus, while depressive symptoms decreased in this comorbid population with CWD-A, significant conduct problem behaviors persisted. The authors concluded that, "acute reductions in depression symptomatology do not affect the course of CD," (p. 664). However, this is not a surprising result given that the intervention did not specifically address conduct problems.

Further rationales for treating conduct problems and depression include 1) the transactional relationship between both disorders ((Lahey, Loeber, Burke, Rathouz, \& McBurnett, 2002, Fergusson et al., 1996) and, (2) the possibility that one disorder may place an individual at risk for developing the second disorder (Capaldi, 1991, 1992; Fergusson et al., 1996, Wolff \& Ollendick, 2006). More specifically, treating one disorder, while neglecting the second disorder, may lead to higher rates of relapse for the treated disorder at subsequent followup and may not affect the untreated disorder at all. For example, one study found that having comorbid CD predicted significantly higher rates of depression relapse during a two-year followup period following psychosocial treatment (Rohde, Clarke, Lewinsohm, Seeley, \& Kaufman, 2001).

In summary, the few studies that have targeted this comorbid population have applied existing protocols shown to be efficacious for only one of the disorders to the treatment of the other disorder. Typically, symptom alleviation in response to these treatments is limited to the targeted disorder. Furthermore, some studies suggest that when the comorbid disorder remains, these youth may be at risk for relapse of the treated disorder (Rohde et al., 2001). To date, no empirically supported treatments have been developed specifically for youth who present with these co-occurring conditions (Ollendick \& King, 2004; Rapp \& Wodorski, 1997). 
The present study, therefore, modified aspects of existing protocols to specifically target symptoms of both conduct problems and depression. In particular, the present study addressed symptoms of both disorders through skills training, family involvement, and cognitivebehavioral techniques. The integrated treatment allows for ongoing attention to both problems, a synthesis of treatment principles, and prioritization of treatment goals.

\section{Treatment Development}

The current manual is based on Social Cognitive Learning Theory (Bandura, 1986). According to this theory, human behavior is based on previous learning, especially the learning of social and interpersonal behavior and of central or core thoughts and beliefs (Carver \& Scheier, 1986). Social behaviors that reflect these learning processes include social communication, problem-solving, and ways of relating to peers and authority figures (Dodge, 1993). Examples of cognitions learned over the course of development include expectancies for control and competence, cognitive biases, and beliefs regarding self, others, and the future (Beck et al., 1979; Rotter, 1996). These learning experiences are based on operant conditioning processes, classical conditioning processes, social reinforcement, and observation and modeling of significant others. Psychopathology among youth is believed to result from faulty learning experiences, reflected in maladaptive cognitions, feelings, and behavior (Carver \& Scheier, 1986). Among adolescents with conduct problems and depression, these maladaptive cognitions and behaviors are reflected in cognitive distortions, coping skill deficits, affect regulation deficits, and problematic social behaviors.

Through CBT, these maladaptive cognitions and behaviors can be addressed. CBT combines behavior change and cognitive information processing methods to modify symptoms of various mental disorders. The separate depression and conduct problem treatment literatures 
provide preliminary evidence for the efficacy of CBT in reducing these problems. Given the high rate of co-occurrence between these disorders and common elements of these treatments, the next logical empirical step is to merge findings from the conduct problem and depression treatment literatures, to create an integrative cognitive behavioral intervention that targets both conditions.

Following review of relevant treatment manuals, a treatment manual was assembled based on empirically supported techniques used in the treatment of both disorders. The TADS manual, a skills oriented treatment based on the notion that depression is either caused by or maintained by depressive thought patterns and the lack of active, positively reinforcing behaviors (TADS Team, 2003), was the primary source. This treatment was augmented by problem solving techniques described by Kazdin $(1987$ a, 1987b, 1992) and Greene $(1998,2005)$ as well as some parent training techniques to improve monitoring and consequences of behavior problems. Parental involvement was also addressed. It should be noted that while the TADS manual included some elements of problem solving and parental involvement, the current manual places greater emphasis on these aspects of treatment. Moreover, the current treatment manual targets symptoms of both disorders simultaneously and on an ongoing basis.

\section{Hypotheses}

The current study aimed to treat both depression and conduct problems in preadolescents. Three main areas of focus were addressed. The first goal was to examine whether the protocol reduced symptoms of conduct problems (i.e., CD or ODD). The second goal was to determine if the intervention effectively reduced symptoms of depression or dysthymia. The third goal was to examine related areas of family functioning that may also improve with reduction of symptoms of conduct problems and depression. Specifically, it was hypothesized that families would 
experience less conflict and more cohesion and expressiveness. These areas were specifically chosen because of the treatment's focus on communication, family activities, and problem solving. Moreover, those with conduct problems and depression tend to have family environments marked by less cohesiveness and greater conflict (George, Herman, \& Ostrander, 2006). Thus, it is predicted that families will experience improvements in these domains as symptoms of these disorders are alleviated. Finally, potential mechanisms of change were explored. Specifically, improvements in emotion regulation and problem solving skills were expected to be the mechanisms of change due to the treatment's focus on these skills. Each of these goals was pursued in a single case design study using non-concurrent multiple baseline design methodology (Carr, 2005).

\section{Methods}

\section{Design}

A non-concurrent multiple-baseline design was implemented with cases beginning treatment after baselines of two, three, or four weeks. This design is essentially a series of A-B replications in which the length of the baseline phase differs in a pre-arranged but random way (Carr, 2005; Christ, 2007). The experimental control demonstrated by the multiple baseline design across participants can be described using three elements of single-case design logic (Cooper, Heron, \& Heward, 1987; Johnston \& Pennypacker, 1993). First, repeated measures can establish the prediction of a baseline's data path into the subsequent treatment phase, allowing the detection of a difference between the actual data path in treatment and the path predicted from baseline. Second, the effects of the independent variable are verified by demonstrating that the intervention changed the participant's behavior. Finally, the effects of the independent variable are replicated across different participants. This design helps to ensure that the 
symptoms of participants are not transient, and changes are not associated with the seeking of treatment or with the assessment process.

\section{Participants}

Participants in this pilot investigation were five youth (3 females, 2 males) and their parents. The children's ages ranged from 11 to 14 years old (mean $=12.6$ years old). Participating youth met criteria for a DSM-IV diagnosis (clinical or subclinical level) of ODD or $\mathrm{CD}$, and a comorbid DSM-IV diagnosis (clinical or subclinical level) of Major Depressive Disorder or Dysthymia. Exclusion criteria included: a) meeting full diagnostic criteria for an autism spectrum disorder, b) current and acute psychotic symptoms, c) estimated IQ less than 80, or d) current suicidal or homicidal ideation. To maximize external validity, youth presenting with other diagnoses were included. Seven children were screened for the current study. One did not meet eligibility criteria and one declined to enter treatment. Of the five children who began treatment, none dropped out of the study. Participant characteristics are summarized in Table 1.

Participant 1, a 14-year-old post-pubescent African-American female, lived with her adoptive parents and biological brother. The family was of upper-middle class income. The participant had a history of physical and sexual abuse and had experienced emotional and behavioral problems since an early age. According to the child and parent DISC (see description of measures below), the participant met criteria for ODD, CD, subclinical major depressive disorder, subclinical dysthymia, mania, and social phobia. In addition, it should be noted that the participant had a past history of molesting her younger brother. She was a student in good academic standing and got along reasonably well with her peers, although her lying and mood difficulties interfered with friendships at times. Her conduct problems and depression were judged as moderate to severe. The participant was not taking any medication at the time of 
referral nor throughout treatment. Based on the Symptom Checklist 90 (SCL-90; see description of measures below) the adoptive parents of this child were not experiencing significant psychological difficulties at the time of referral.

Participant 2 was a pre-pubescent 11-year-old Caucasian male who met criteria for ODD, $\mathrm{CD}$, subclinical major depressive disorder, attention deficit-hyperactivity disorder, separation anxiety, panic disorder, and mania on the DISC. He resided with his biological mother and two younger half-siblings. He had never had contact with his biological father. His step-father was not in the home on a regular basis, his mother was unemployed, and the family was of low income. The participant had been hospitalized on two occasions due to mood problems and a suicide attempt. At pretreatment, he was taking Seroquel, Focalin, Clonidine, and Zoloft. He remained on these medications throughout treatment. He was doing well academically despite frequent removals from the classroom due to behavior problems. Socially, the participant had considerable difficulty and his only friends were two relatively mute classmates. He was often bullied and was not accepted by his peers. His conduct problems and depression were determined to be markedly severe. At intake, his mother indicated on the SCL-90 that she was experiencing symptoms of anxiety $(\mathrm{T}=65)$.

Participant 3, a post-pubescent 11-year-old Caucasian female, met DISC criteria for ODD, major depressive disorder, and separation anxiety. She lived with her biological parents and younger brother. The family was of low-middle income and was experiencing financial difficulties. Academically, the participant did well and she had an average number of friends, although she rarely engaged in social activities outside of school. She was not taking any medication at the time of referral nor throughout treatment and her conduct problems and depression were determined to be markedly severe. The participant's mother reported on the 
SCL-90 that she was experiencing interpersonal sensitivity $(\mathrm{T}=67)$, depression $(\mathrm{T}=66)$, and paranoid ideation $(\mathrm{T}=69)$.

Participant 4 was a post-pubescent 13-year-old Caucasian female who resided with her biological parents and younger sister. The family was of middle-income status. Based on the DISC interview, the participant met criteria for CD and subclinical major depressive disorder. She also engaged in self-mutilating behaviors (i.e., cutting) on a few occasions. She received low to average grades and had an average number of friends. She was not taking medication at the time of referral nor throughout treatment and her conduct problems and depression were determined to be mildly severe. No parental psychopathology was reported on the SCL-90.

Finally, Participant 5 was a post-pubescent 14-year-old Caucasian male. At pretreatment he met criteria for ODD, CD, subclinical major depressive disorder, subclinical dysthymia, social phobia, and attention deficit-hyperactivity disorder as established by the parent and child DISC. The participant lived with his biological mother, step-father, and three younger half-siblings. He had occasional contact with his biological father. The family was of middle income. The participant was enrolled in the gifted program and received average grades. He tended to be liked by his peers; however, he occasionally got in fights at school. Based on the DISC and the initial clinical interview, his conduct problems and depression were judged as marked severity. At the time of referral, he received Risperdal and Zoloft that he continued taking these throughout treatment. No parental psychopathology was endorsed on the SCL-90.

\section{Measures}

Diagnostic Interview Schedule for Children (DISC; Shaffer, Fisher, Lucas, Dulcan, \& Schwab-Stone, 2000). The computerized DISC is a highly structured diagnostic assessment instrument designed to gather symptom presence for 34 child and adolescent psychiatric 
disorders (including mood disorders and conduct problems). It is currently one of the most widely used child and adolescent structured interviews (Shaffer et al., 1996). The DISC was originally designed to be administered by trained lay interviewers to aide in gathering diagnostic information for large-scale epidemiological studies. However, it has been used in numerous clinical research trials (Friman et al., 2000) and was used in the formulation of the DSM-IV criteria for these disorders (Frick et al., 1994; Lahey et al., 1994). The current version of the DISC is composed of questions based on symptom and criterion variables as defined in the DSM-IV. The reliability and validity of the DISC have been assessed through a variety of methods (Shaffer et al, 1996). Schwab-Stone et al. (1996) estimated the test-retest reliability of an earlier version of the DISC at .56. In comparison samples with earlier versions of the DISC, the current version compares favorably (Shaffer et al., 1996). Previous versions of the DISC have demonstrated at least adequate concurrent validity when comparing the DISC to clinicians' diagnoses (Shaffer et al., 1996). The computerized DISC is administered via a computer (the interviewer reads questions from the computer and enters the responses directly in the computer) and scored by computer algorithms. There are two parallel versions, one for youth and one for parents or primary caretakers. The interview lasts approximately $1-2$ hours depending on the child's psychological difficulties. This measure was used to assess DSM-IV diagnoses. Subclinical major depressive disorder, dysthymia, ODD, and CD were defined as an insufficient number of symptoms or a sufficient number of symptoms but insufficient duration. Specifically, subclinical depression cases were defined as adolescents who reported at least two but less than five symptoms from the major depressive disorder symptom criteria. At least one of the symptoms had to be depressed mood, irritability or loss of interest; the symptoms had to be present during the same two-week period and represent a significant change from a previous 
level of functioning. Subclinical depression cases also included those individuals who endorsed five or more clinically significant symptoms, but indicated that these symptoms did not persist for a full two weeks. Bereavement was used as exclusionary criteria. For subclinical dysthymia, participants endorsed irritable mood plus one other symptom that persisted for a year or endorsed the required number of symptoms, but indicated that they persisted less than 1 year, but more than six months. Bereavement was again used as exclusionary criteria. For ODD and CD, participants had to endorse at least half of the required symptoms for the specified period of time (i.e., 6 months).

The "OR rule" was used to combine parent and child reports, with each disorder considered present if reported by either the parent or child, as recommended by Piacentini et al. (1992). However, it should be noted that there was considerable disagreement between the parent and child DISC reports in the present study. Agreement was found in only one instance with Participant 2 and his mother agreeing on a diagnosis of ODD at pre-treatment. For all other participants, conduct problem and depression diagnoses were endorsed by the parent but not the child.

Conduct Problem and Depression Rating Scale. This measure was designed for the purposes of the present study. The measure includes symptoms of ODD and depression drawn from the DSM-IV and rated for the frequency of the behavior. In addition, a checklist for symptoms of $\mathrm{CD}$ was included. This measure was administered to mothers and adolescents on a weekly basis during baseline and the active treatment phase as well as at 1-month follow-up.

Child Behavior Checklist (CBCL; Achenbach, 2001a). The CBCL is a 113-item survey assessing competencies and problems of children. The mother reported how true each statement was of their adolescent on a scale of 0 ('not true, as far as you know'), 1 ('somewhat or 
sometimes true'), and 2 ('very true or often true'). The CBCL addresses the child's sleep problems, aggressive behavior, internalizing and externalizing problems, depressive symptoms, hyperactivity, anxiety, withdrawn behavior, as well as other problem behaviors. The CBCL also includes three social competence scales: Participation (child participation in activities), Social (child interactions with others), and School (child's academic performance). The CBCL evidences high reliability ranging from .85 to .96 . CBCL scores served as an additional measure for the adolescent's symptoms of conduct problems and mood disorders as well as current aggression and withdrawal. This measure was administered at pre-treatment, post-treatment, and 1-month follow-up.

Teacher Report Form. (TRF; Achenbach, 2001b). The teacher rating form is a questionnaire that includes 113 items to which the teacher is asked to indicate if each behavior/characteristic is often/always true, not true, or sometimes true of the student being assessed. Test-retest reliability over a 15-day period is .90 for adaptive behavior scales and .95 for problem behavior scales.

Youth Self Report (YSR; Achenbach, 2001c). This instrument is a standardized selfreport which assesses competency and problem based areas in youth ages 11 to 18 . The YSR consists of 112 items, both open-ended (e.g., "Please describe the best things about yourself.") and self-rated (e.g., "I get in many fights"; $0=$ Not true, $1=$ Somewhat or sometimes true, $2=$ Very often true), yielding scores for a variety of scales, including social competency, externalizing and internalizing, aggressive behaviors, and DSM-based scales (Achenbach, 1991; 2001c). Reliability coefficients for the YSR have ranged from .67 to .88. YSR reports served as an additional measure for conduct problem and mood disorder diagnoses as well as a measure for 
aggression and withdrawal. This measure was administered at pre-treatment, post-treatment, and 1-month follow-up.

Child Depression Inventory (CDI; Kovacs, 1992). The CDI is a 27-item paper and pencil questionnaire. For each item, the child was presented with three statements representing varying levels of symptomatology. The child was asked to choose the statement that best described him/her. Adequate reliability for the CDI for both boys and girls across several age groups (coefficient alphas ranging from .83 to .89) has been established (Smucker, Craighead, Craighead, and Green, 1986). This measure was administered at pre-treatment, post-treatment, and 1-month follow-up. Mothers also completed a parallel version of the form on their adolescent son or daughter at pre-treatment, post-treatment and at 1-month post-treatment.

Disruptive Behavior Disorders Rating Scale (DBD; Barkley, 1997; Pelham, Gnagy, \& Greenslade, 1992), The DBD is comprised of the DSM-IV symptom lists for ADHD, ODD, and $\mathrm{CD}$ and uses a 4-point response scale ranging from 0 (not at all) to 3 (very much). The DBD has been shown to have excellent psychometric properties (see Pelham et al., 1992, for normative data). This measure was administered to mothers at pre-treatment, post-treatment and at 1-month post-treatment. In addition, adolescents completed a parallel version of the form at pre-treatment, post-treatment and at 1-month post-treatment. For the present study, the Barkley (1997) revision of the scale was used and only ratings of ODD and CD symptoms were obtained.

Clinical Global Impression-Severity and Improvement Scales (CGI-S; CGI-I; Guy, 1976). The CGI-S and CGI-I assess severity and improvement of the child's conduct problem behavior and depression on a 7-point scale, with lower scores indicating less severity and more improvement, respectively. The CGI-S and CGI-I was completed by the therapist on a weekly 
basis during active treatment and during designated follow-up intervals. The scales have been used successfully in a number of randomized clinical trials for child psychopathology.

Consumer Satisfaction Questionnaire (CSQ). Based on the Consumer Satisfaction Scale (CSS; McMahon \& Forehand, 2003), the CSQ was designed to assess parental and child satisfaction with the treatment approach and the strategies they use in terms of both difficulty and usefulness. Mothers and children are asked to rate each of 11 items along a seven-point scale. Five of the items are open-ended questions regarding the family's experiences with the program. Mothers were asked to complete this measure at weeks 4 and 7, at post-treatment, and at 1-month post-treatment.

Symptom Checklist - 90-R (SCL-90-R; Derogatis et al., 1994). The SCL-90-R is a 90item questionnaire that describes symptoms of general psychological distress experienced by mothers. For each item the subject is asked to rate severity over the past week. Responses are made on a five-point scale ranging from "not at all" to "extremely." The SCL-90-R contains three global indices and 10 symptom scales. Adequate internal consistency for the scales has been documented. This measure was administered at pre-treatment only.

Family Environment Scale (FES; Moos \& Moos, 1981). The FES is a 90-item, true/false, self-report measure that assesses various characteristics of family relationships. This measure contains ten subscales ( 9 items each) that are divided into three dimensions designed to assess socio-environmental aspects of family functioning. The Relationship dimension of the measure was used for this study and includes measurements of Cohesion, Expressiveness, and Conflict. The reliability and validity of the FES are well-established (Moos \& Moos, 1981). Internal consistency reliability estimates for the FES subscales range from .61 to .78. Test-retest reliabilities for the subscales for 2-month, 3-month, and 12-month intervals range from .52 to .91 
suggesting that the scale is reasonably stable across time. This measure was administered at pretreatment, post-treatment, and 1-month follow-up.

Vocabulary and Block Design Subtests from the Wechsler Intelligence Scale for Children-IV (WISC-IV; Wechsler, 2003). Cognitive ability was estimated using age-corrected scores on the Block Design and Vocabulary subtests from the WISC-IV. These two subtests are frequently used to estimate Full Scale IQ. They correlate .90 with the Full Scale IQ (Wechsler, 2003). The WISC-IV is appropriate for youth between 6 and 16 years of age.

Pubertal Development Scale (PDS; Petersen, Crockett, Richards, \& Boxer, 1988). The PDS is an 8-item measure that assesses the child's current stage of pubertal development. This self-report instrument was designed to be used by adolescents to report development on five indices of pubertal growth using a 4-point scale. The questionnaire asks about growth, body hair, and skin changes for all participants. Males are also asked about changes to voice and growth of facial hair and females are asked about breast development and menarche. Internal consistency alphas for males and females have been reported above .75 , indicating acceptable reliability (Ge et al, 2006). In addition, this self-report measure is significantly correlated with other more objective measures of pubertal status, such as physician's ratings on the Tanner stage $(r=.61$ to .67; Brooks-Gunn, Warren, Rosso, \& Gargiulo, 1987) or interviewer's ratings ( $r=.70$; Peterson et al., 1988). This questionnaire was administered at pre-treatment only.

Emotion Regulation Checklist (ERC; Shields \& Cicchetti, 1997). The ERC is a 24-item mother-report measure of a child's Lability/Negativity and Emotion Regulation. Frequency of affective behaviors are rated on a 4-point scale ranging from 1 (Never) to 4 (Almost Always). The Lability/Negativity subscale is comprised of items representing a lack of flexibility, mood lability, and dysregulated negative affect; sample items include "Exhibits wide mood swings," 
and "Is prone to angry outbursts." The Emotion Regulation subscale includes items describing situationally appropriate affective displays, empathy, and emotional self-awareness; sample items include "Is empathic toward others," and "Can say when s/he is feeling sad, angry or mad, fearful or afraid." Cronbach's $\alpha$ for this measure have been found to be .83 to .96 (Shields \& Cicchetti, 1997). This measure was administered at pre-treatment, post-treatment, and 1-month follow-up. This measure was used to index changes in emotion regulation and as one of the measures of mediation thought to be central to the intervention.

Problem Solving Discussion Task and Rating Scale (Oregon Social Learning Center, 1991). The problem-solving task begins with the parent completing a Parent Issues Checklists that provides a list of 27 issues (e.g., going to bed on time, fighting with siblings, talking back, etc.) that are often problematic for parents and children. The parent chooses one of these issues to discuss during the problem solving activity. The parent(s) and child are then asked to discuss this problem for a period of 7 minutes and to try to come up with a solution (or solutions) to the problem. These interactions were videotaped and trained research assistants rated the interactions based on the participants' communication skills, positive and negative involvement, and ability to propose and agree on appropriate solutions to the problem in accordance with manualized coding procedures. Inter-rater agreement analyses for the current study indicated moderate agreement $(\kappa=.79)$.

In addition, the parent and child completed a questionnaire regarding their own impressions of their problem-solving interaction. The Problem Solving Discussion Rating Scale consists of six questions pertaining to problem-solving issues within the family. For each of the six questions, the parent and child independently rated the issues on a 5-point scale. It too was 
used as a measure of potential mediation for the treatment and was administered at pre-treatment, weeks 5 and 8, post-treatment, as well as at 1-month follow-up.

\section{Procedure}

Participants were recruited from the Virginia Tech community by contacting mental health professionals, centers providing assessment and treatment services to youth, and schools. In addition, children who received a diagnosis of conduct problems and depression through the Assessment Clinic at the Child Study Center of Virginia Tech were contacted. A two-stage ascertainment procedure was used. First, parents of children expressing interest in the study completed a telephone interview to screen for ODD, CD, Major Depressive Disorder, and Dysthymia. Parents whose children appeared to meet initial eligibility criteria were then scheduled for a baseline comprehensive assessment to confirm diagnoses and suitability for the treatment protocol. Written informed consent was obtained from one parent or caregiver and assent from the adolescent. Participants were also notified that they could withdraw from the study at any time (see Appendix).

At the baseline assessment, the child and parent computerized DISC interviews were administered by trained undergraduate research assistants, trained to requisite levels of competence to help ensure reliability and validity of data procurement. In addition, the child completed sections of the WISC-IV to estimate IQ level and several self-report measures. Mothers completed measures pertaining to the child's behaviors, the family environment, and the mothers' symptoms of psychopathology. Finally, the child and parent participated in the problem solving task and completed a questionnaires about this interaction. At post-treatment and 1month follow-up, the sections of the DISC for which the child met diagnostic criteria at the 
baseline period were re-administered. The assessment measures given at pretreatment were also administered at post-treatment and 1-month follow-up.

Those children who met eligibility criteria and their parents were randomly assigned to baseline phases lasting two, three, or four weeks (two participants in each condition except the four week condition which had only one participant). During each week of baseline, adolescents and their mothers were asked to complete a questionnaire on a weekly basis to establish data trends prior to intervention. Following the baseline period, the intervention phase began (outlined below). Repeated observations continued as during baseline to measure what changes occurred in the dependent variables (Harvey, May, \& Kennedy, 2004). In addition to the weekly measures, adolescents and their mothers also completed the problem solving task in weeks 5 and 8 and the Consumer Satisfaction Survey. The clinician completed the CGI-S/I each week.

Treatment. Treatment involved 11 to 16 weekly sessions which were generally one hour in length, but 90 minutes was allotted for sessions involving greater parent involvement. As mentioned previously, this cognitive behavior therapy manual was developed by modifying and integrating modules from skills-based treatments for depression and behavior problems. The manual includes an explicit description of the goals and content for each session, and standard handouts for many sessions are provided. All sessions include common components including review of previous week events, review of homework, skill introduction and practice, homework assignment, and a parent "check-in." There is a menu of sessions to choose from and sessions may be repeated if deemed necessary. This relatively flexible format allows for tailoring to individual treatment needs. The manual includes modules that employ problem solving, cognitive restructuring, and affect regulation techniques designed to address thoughts, feelings, and behaviors specifically associated with depression and conduct problems. More specifically, 
session content consists of the following major components: (a) psychoeducation, (b) mood monitoring, (c) goal setting, (d) reinforcing activities, (e) affect identification and regulation, (f) problem solving, (g) cognitive restructuring, (h) communication, (i) social skills training, and (j) relapse prevention.

Therapist. All participants were treated by the current author who was a masters-level graduate clinician enrolled in an APA approved doctoral program in clinical psychology. The clinician was supervised by a licensed clinical psychologist and the protocol was implemented in a clinically sensitive and flexible manner.

Competency. To assess therapist competency, all sessions were videotaped and rated by two separate coders on a 5-point scale (0 to 4) with higher scores indicating greater competency. Rater 1 endorsed a mean rating of 3.90 and Rater 2 indicated a mean score of 3.96. Averaging across these two raters resulted in a competency score of 3.93, suggesting a high level of therapeutic skill. There was $93 \%$ agreement between raters.

Treatment Adherence. Two separate coders rated all videotaped sessions to assess the degree of treatment adherence. The tapes were rated based on the degree of adherence to various elements of the treatment approach that were the focus of individual sessions. The scale consisted of 23 items that were rated on a 5-point scale (0 to 4) with lower scores indicating poorer adherence to the protocol. Rater 1 indicated a mean adherence score of 3.73 and Rater 2 indicated a mean score of 3. 67. An overall adherence rating of 3.70 was calculated, reflecting a high degree of adherence. There was $80 \%$ agreement between raters.

Statistical Analyses. Given the small sample size, statistical analyses were necessarily limited. One of the more common methods for evaluating multiple baseline studies is through visual inspection and noting whether gains were made during the intervention (Carr, 1995). 
Differences between baseline and the treatment phase are evaluated for differences in the mean, (e.g., average frequency of symptoms) level (e.g., whether the change was stable), changes in the trend, or slope (either increase or decrease) of the data, and the latency of change (how quickly treatment gains were observed) (Kazdin, 1998). Intervention effects are believed to be more clear (i.e., fewer extraneous influences) if the change in behavior occurs close in time to the beginning of the treatment phase (Kazdin, 1998).

Beyond visual inspection, exploratory analyses were conducted in order to determine the effects of treatment. Paired t-tests were used to compare pre-treatment and post-treatment scores. Such analyses have been recommended for studies using multiple baseline designs (Kazdin, 1982; Kazdin, 1998; Richards, Taylor, Ramasamy, \& Richards, 1999). A Bonferroni correction was considered, but because of the potential clinical significance of this pilot study, a greater chance of Type I error was allowed so that potentially important findings would not be dismissed.

Results

Weekly Ratings of ODD Symptoms. As can be seen in Figure 1, the frequency of ODD symptoms decreased for the majority of participants. However, response to treatment was not consistent across participants and some did not show improvements. Furthermore, considerable variation was shown from week to week suggesting that participants experienced both increases and decreases in their symptom frequency over the course of treatment. In addition, 1-month follow-up scores for some participants showed an increase in symptoms from post-treatment suggesting that changes in symptoms of oppositionality were not maintained over time. As is commonly reported in the literature, youth self-report scores of oppositional symptoms tended to be lower than maternal reports as is common in the literature (see Collett, Ohan, \& Myers, 2003). 
Table 2 shows mean baseline scores as well as post-treatment and 1-month follow-up scores. According to these scores, mothers of Participants 1, 4, and 5 reported improvements from pre-treatment to post-treatment. However, this change was not stable as mothers of Participants 4 and 5 reported an increase in symptoms at 1-month follow-up. Based on selfreports, Participants 1, 3, and 4 had a reduction in symptoms from mean baseline scores to posttreatment. At 1-month follow-up improvements were evident for Participants 3, 4, and 5. This suggests that the change was not stable for Participant 1; however, Participant 5 continued to improve as his self-reported symptoms dropped to 0 .

Consistent with single case methodology, an examination of individual weekly symptom frequencies was undertaken. Participant 1 had considerable variation in oppositional defiant symptom frequency during the baseline period. At week 4 of the baseline, symptom frequency was on an upward trend according to both raters suggesting that symptoms worsened without treatment intervention. However, symptoms continued to increase during the first two weeks of treatment before beginning to decrease in week 3 (according to mother and child). Symptoms continued to fluctuate and both raters reported an increase again in week 9. This change corresponded with the time the participant received poor grades and consequently was placed on probation from her middle school basketball team. However, following that point, symptoms generally decreased. At week 15, symptom frequency was on a downward trend according to maternal and adolescent reports and a considerable reduction from baseline to week 15 and into post-treatment was observed. Thus, the participant showed significant improvement from baseline and appeared to benefit from treatment. However, this change was not stable since at 1month follow-up maternal and adolescent ratings of symptoms had increased. 
For Participant 2, maternal and youth ODD symptom frequency ratings remained stable throughout each phase with only slight variations. His scores remained near baseline ratings and did not show significant fluctuation as a result of treatment implementation. In addition, his posttreatment and 1-month follow-up ratings were similar to baseline scores, further suggesting a lack of improvement. However, it should be noted that the baseline symptom frequency for this participant was low and thus, there was little room for improvement based on this measure. It is notable that both maternal and adolescent ratings were consistent despite weekly fluctuations in behavior. For example, in the beginning of treatment, the student was suspended from school for a fight and at the end of treatment he was nominated as "Student of the Month."

For Participant 3, maternal and adolescent ratings of ODD symptoms were somewhat discrepant. According to maternal ratings, ODD symptoms were stable through the baseline phase and the first 7 weeks of treatment. In week 8, a considerable drop in symptom frequency was observed and corresponded with her transferring to another school. Over the next several weeks, some variation in ratings was observed and by the end of treatment, maternal ratings of symptoms were only slightly below baseline reports. These ratings remained consistent at posttreatment and 1-month follow-up. Participant 3's self-report of symptoms showed a considerable downward trend during the baseline period followed by fluctuations in weeks 1 through 8 (initially an upward trend followed by a downward trend). Symptoms rated by this participant reached a low in week 9 and then generally increased through the end of treatment. However ratings at post-treatment and 1-month follow-up suggest improvements from the mean baseline rating. Thus, both the maternal and youth ratings suggest some improvement according to post-treatment and 1-month follow-up ratings in comparison to the mean baseline ratings. 
Participant 4 showed a slight downward trend, according to both maternal and adolescent reports during the baseline period that continued through the first 2 weeks of treatment. The Participant then showed some variation in symptom frequency before reaching a score of 0 , according to both mother and youth, in week 9. From week 9 through the end of treatment a general upward trend was observed, but post-treatment symptom ratings remained below baseline ratings. Ratings at 1 -month follow-up showed a slight increase from post-treatment scores with maternal and youth symptom ratings falling slightly below mean baseline reports. It should again be noted that because baseline ratings for this participant were low, there was less room for improvement.

Finally, Participant 5 maternal and adolescent ratings showed some discrepancies. According to the participant's mother, baseline ratings showed an upward trend suggesting worsening symptom frequency, but symptoms leveled out and began a downward trend with treatment implementation. Weeks 4 through 9 showed some variation (increased, followed by decrease, and another increase). It should be noted that during this period of time, there were higher levels of parent-child conflict and the child ran away from home. Despite these difficult times, treatment ended with a downward trend of symptoms suggesting improvement. Moreover, post-treatment and 1-month follow-up maternal ratings were significantly below baseline scores. According to Participant 5's self-report, symptom frequency was low but stable during the baseline period. When treatment was implemented a downward trend was observed with a rating of 0 symptoms by week 2 . However, the child then reported an increase in symptom frequency through week 5 , followed by a decrease in symptoms through the remainder of treatment. The participant rated oppositional defiant symptoms a 0 in weeks 10 and 11 . However, post-treatment self-report ratings were again higher and did not differ from baseline. At 1-month follow-up, the 
participant's symptom frequency had returned to 0 suggesting improvement. In all, Participant 5's maternal report suggests significant improvement that was maintained at 1-month follow-up while self-report suggests slight improvement over at 1-month follow-up, but not at posttreatment.

Weekly Ratings of Depression Symptoms Weekly ratings of depression show similar patterns. As can be seen in Figure 2, the frequency of depression symptoms decreased for the majority of participants according to maternal and child reports. However, participants showed week to week variations with both increases and decreases in symptom frequency. In a comparison of mean baseline scores to post-treatment scores (Table 2) it is apparent that mothers of Participants 1, 3, 4, and 5 reported improvements from baseline to post-treatment and these improvements were maintained at 1-month follow-up. Based on self-reports, Participants 1, 3, and 4 had a reduction in symptoms from mean baseline scores to post-treatment. At 1-month follow-up, scores were similar to post-treatment ratings for four of the five participants. However, Participant 1's self report indicated that symptoms of depression had increased to a level consistent with her baseline ratings. Conversely, the maternal report for Participant 1 indicated that symptoms continued to decrease from post-treatment to 1-month follow-up. Overall, however, treatment gains related to symptoms of depression tended to be maintained at 1-month follow-up. A specific look at each participant's weekly ratings follows (see Figure 2).

Participant 1's frequency of depression symptoms showed considerable variation during the 4 week baseline period and the mother and child reported a downward trend at the end of the baseline phase. This downward trend may indicate that the participant would have improved without intervention, but the wide variation in baseline ratings makes it difficult to draw firm conclusions. According to maternal report, the frequency of depression symptoms decreased 
with treatment implementation and remained level through week 9. In week 10, a strong upward trend was observed (corresponding with the participant's dismissal from the basketball team) followed by a general downward trend through the end of treatment. Post-treatment ratings were significantly below baseline scores and remained low at 1-month post-treatment. The participant's self report is mostly similar, with the exception of an increase in symptom frequency during week 2. The participant rated depression symptoms lower than baseline symptoms at post-treatment, but reported that symptoms had increased by 1-month follow-up. In all, the participant showed significant improvement from baseline to post-treatment according to both mother and child. However, 1-month follow-up ratings are discrepant with the mother reporting stable symptom frequency and her daughter reporting an increase in symptoms. Thus, the stability of the improvement for this participant is inconclusive based on these ratings.

Participant 2 demonstrated few improvements from baseline to post-treatment and 1month follow-up and only slight variations in depression symptom frequency were observed. Maternal ratings remained fairly level through baseline and treatment implementation. Posttreatment and 1-month follow-up ratings were only slightly below baseline scores. The participant's self-report of baseline depression symptom frequency ended in a downward trend, but the scores were equivalent for weeks 1 and 3 of baseline. The last week of treatment showed a downward trend, but post-treatment and 1-month follow-up ratings were similar to baseline ratings. In sum, this participant's symptoms remained fairly stable as a result of treatment.

Participant 3's maternal and self-report ratings showed some discrepancy. Both mother and adolescent reported a downward trend in symptoms from week 1 to week 2 of baseline, suggesting that symptoms may have improved without treatment. Maternal ratings showed that this decrease in symptom frequency generally continued over the course of treatment (with an 
exception at week 11). Post-treatment and 1-month follow-up scores were below baseline ratings, suggesting that the participant benefited from treatment. The participant's self-report showed wider variation over the course of treatment with upward and downward trends observed from week to week. However, treatment ended on a downward trend. Moreover, post-treatment and 1-month follow-up scores were considerably lower than the mean baseline score. Thus, this participant seems to have benefited from treatment in terms of the frequency of depression symptoms.

For Participant 4, mother and adolescent tended to agree on ratings of depression. However, during the baseline period, the mother reported a slight upward trend in symptom frequency while the child reported a slight downward trend. Still, maternal and youth ratings were within a few points of one another. Both reported an initial increase in symptom frequency at week 2 which was alleviated by week 3 and remained level through the end of treatment. The increase in week 2 corresponded with the participant's break up with a boyfriend and engagement in cutting behavior. Post-treatment and 1-month follow-up ratings were only slightly lower than baseline ratings. However, the frequency of depression symptoms was low during the baseline period, limiting room for improvement.

Finally, for Participant 5, maternal and youth ratings showed a downward trend during the baseline period indicating some symptom improvement without intervention. Symptom frequency showed some variation for the first half of treatment, but a general upward trend was observed indicating that symptoms became more frequent during this time. During the second half of treatment, maternal and adolescent symptom ratings showed a downward trend. At posttreatment and 1-month follow-up, maternal ratings indicated a decrease in symptoms from baseline whereas child ratings were similar to baseline levels. 
Diagnostic Status. Table 3 presents the diagnostic status of the five participating youth at pre-treatment, post-treatment, and 1-month follow-up. Treatment gains were evident in diagnostic status changes across all participants. At post-treatment, Participant 2 no longer met criteria for ODD or CD while the other four participants met criteria for ODD, but not CD. All five children failed to meet criteria for MDD or dysthymia, but three children (Participants 1, 3, and 4) met subclinical criteria for MDD or dysthymia. Comparing pre-treatment status to posttreatment status, four participants (Participants 1,2, 4, and 5) experienced positive change in conduct problem diagnoses (ODD or CD) and four participants (Participants 1, 2, 3, and 5) experienced positive change in diagnoses of depressive disorders (MDD or dysthymia). At 1month follow-up, two children (Participants 2 and 4) did not meet full criteria for ODD or CD, but met the subclinical criteria; all five children did not meet full criteria for MDD or dysthymia, but two children (Participants 3 and 4) met subclinical criteria. In addition, three of the four youth who presented with other comorbid diagnoses at pre-treatment, experienced additional changes in diagnostic status at post-treatment and 1-month follow-up. Specifically, Participant 1 no longer met criteria for mania or social phobia; Participant 3 did not meet criteria for separation anxiety disorder; and Participant 5 failed to meet criteria for ADHD or social phobia.

Symptomatology. Table 4 presents maternal-, teacher-, and child-reports of conduct problem and depression related symptomatology (CBCL, TRF, YSR). It is notable that the majority of pre-treatment scores, as reported by mothers, teachers, and youth, were below the clinical cutoff, providing little room for improvement.

According to mothers, Anxious/Depressed and Withdrawn/Depressed symptoms were reduced for four of the five participants (Participants 1, 2, 3, and 5) from pre- to post-treatment and Rule Breaking and Aggressive Symptoms were reduced for three of the five participants 
(Participants 1, 4, and 5). Average reductions in scores from pre- to post-treatment were -4.8 for Anxious/Depressed, -6.4 for Withdrawn/Depressed, -3.2 for Rule Breaking, and -7.2 for Aggression. These reductions were generally maintained at 1-month follow-up. Mean changes from post-treatment to 1-month follow-up were as follows: Anxious/Depressed $=-3.6$, Withdrawn/Depressed $=-3.8$, Rule Breaking $=-2.4$, and Aggression $=-3.8$.

Based on TRF scores at pre- and post-treatment Anxious/Depressed symptoms increased for Participants 1 and 5, but decreased for the other three participants. Withdrawn/Depressed symptoms increased for Participants 1 and 5, decreased for Participants 2 and 3, and remained equal for Participant 4. Rule Breaking increased for Participants 4 and 5 and decreased for all others. Aggression increased for four participants but decreased for Participant 2. Mean changes in scores from pre- to post-treatment were -4.4 for Anxious/Depressed, -3.4 for Withdrawn/Depressed, 4.0 for Rule Breaking, and 0.0 for Aggression. Thus, on average participants showed improvements on the two internalizing subscales but their symptoms remained the same or worsened on the externalizing subscales.

From post-treatment to 1-month follow-up, teacher reports on the TRF indicated that participants' internalizing symptoms (Anxious/Depressed and Withdrawn/Depressed subscales) tended to increase while Rule Breaking showed a slight decline and Aggressive behavior showed a slight increase (mean change for Anxious/Depressed $=7.6$, mean change for Withdrawn/Depressed $=3$, mean change for Rule Breaking $=-3.0$, and mean change for Aggression $=0.8$ ). Looking at individual participants, Participant 1 remained the same from post-treatment to 1-month follow-up, Participants 2 and 5 showed further decreases on three of the four scales, Participant 3 increased on all four scales, and Participant 4 increased on three of 
the four scales. Thus, three of the five participants maintained their therapeutic gains at 1-month follow-up.

Based on the YSR, self reported symptoms generally showed equivocal results from preto post-treatment although minor differences were observed. Anxious/Depressed symptoms increased by 1 point for Participant 5, decreased for Participants 1 and 2, and were equal from pre- to post-treatment for the remaining participants. Withdrawn/Depressed symptoms increased for Participants 1 and 5, decreased for Participants 2 and 4, and remained the same for Participant 3. Rule Breaking decreased for Participants 1, 2, and 4 and remained the same for the others. Aggression increased for Participant 5, decreased for Participants 1, 2, and 4, and remained the same for Participant 3. Mean changes in YSR scores across participants from pretreatment to post-treatment were -2.6 for Anxious/Depressed, -2.0 for Withdrawn/Depressed, 2.4 for Rule Breaking, and -5.4 for Aggression. Few changes were observed on the YSR from post-treatment to 1-month follow-up. Mean changes in scores from post-treatment to 1-month follow-up were -2.6 for Anxious/Depressed, -0.4 for Withdrawn/Depressed, -1.2 for Rule Breaking, and -.06 for Aggression.

Table 5 presents mother and youth ratings of symptoms related to depression and conduct problems based on the CDI and DBDRS. It should be noted that at pre-treatment, only Participant 1's mother endorsed clinically elevated symptoms of depression. All other mother and adolescent CDI ratings were in the non-clinical range at pre-treatment. In terms of conduct problem symptoms, all five mothers and 1 child (Participant 2) reported symptoms of ODD in the clinical range. Three children reported a score of 0 for ODD symptoms. Finally, three mothers (Participants 1, 3, and 5) endorsed symptoms of CD in the clinical range. No self-reports of CD symptoms were in the clinical range. 
At post-treatment, Participants 3, 4, and 5 showed decreases in mother endorsed symptoms of depression on the CDI whereas self-reports of depression symptoms suggested decreases for Participants 1,3 , and 4 . (mean change $=0$ for mothers and -1.0 for children). However, maternal ratings for Participants 1 and 2 and child ratings for Participants 2 and 5 suggested increases in depression. At post-treatment, clinically elevated depression scores were evident in maternal ratings for Participants 1 and 2 and the self-report for Participant 2.

A reduction in ODD symptoms on the DBDRS was endorsed by mothers of all five participants (mean change $=-4.8$ ). In addition, Participants 2 and 3 self-reported symptom reductions whereas the other three participants consistently endorsed 0 symptoms (mean change $=-0.8$ ). In terms of CD symptoms, mothers of Participants 1, 3, 4, and 5 reported a decrease in symptoms (mean change $=-2.8$ ). At post-treatment, only Participant 2 remained in the clinical range for ODD symptoms according to maternal report. No participants were in the clinical range for $\mathrm{CD}$ symptoms

At 1-month follow-up, mothers of Participants 1, 2, 3, and 4 reported decreases in symptoms of depression from post-treatment. Self-reports on the CDI at 1-month follow-up were generally equivalent to post-treatment scores although minor additional improvements were evident for Participants 1, 4, and 5. In terms of ODD symptoms, mothers of Participants 1, 3, and 5 reported increases (ranging from 1 to 5 points) from post-treatment to 1-month follow-up while mothers of Participants 2 and 4 reported that symptoms were better or the same as post-treatment scores. In terms of ODD symptoms, mothers of Participants 2 and 4 reported that treatment gains were maintained where as slight increases in symptoms were endorsed for Participants 1, 3, and 5. Self-reported ODD symptoms showed further improvements for Participants 2 and 3 while the other participants consistently endorsed zero symptoms of ODD. Finally for CD symptoms, 
mothers of Participants 1, 2, and 4 reported that symptoms were the same or better than at posttreatment. However, mothers of Participants 3 and 5 reported increases in symptoms from posttreatment. Thus, three of the five participants maintained their improvements at 1-month follow up for CD symptoms.

Emotion Regulation and Family Environment. Table 6 presents maternal ratings for the ERC and FES. On the Emotion Regulation Checklist, Lability/Negativity remained equal for Participant 3 from pre-treatment to post-treatment, but improved for the other four participants (mean change $=-3.8)$. Similarly, Emotion Regulation was rated higher at post-treatment for Participants 1, 2, 3, and 5, but remained equal for Participant 4 (mean change $=2.2$ ) indicating that on average participants showed slight improvement. At 1-month follow-up, mothers of Participants 1, 3, and 4 reported slight increases in Lability/Nagativity from post-treatment while mothers of Participants 2 and 5 reported that gains were maintained or improved further (mean change $=-0.04)$. For Emotion Regulation, slight variations were reported such that Participants 1, 2, and 5 had slight increases, and Participants 3 and 4 had slight decreases (mean change $=-$ $1.0)$.

Ratings from pre-treatment to post-treatment suggest improvements in the family environment. Specifically, on the Conflict subscale of the FES, Participants 1, 2, and 5 endorsed improvements (decreases), Participant 3 endorsed an increase, and Participant 4 reported that conflict in the home remained the same (mean increase $=8.8$ ). On the Cohesion subscale, all five participants indicated an increase in family cohesion (mean increase $=13.2$ ). Finally, on the Expressiveness subscale, Participants 1, 2, and 5 indicated improvements (increase), Participant 4 reported a decrease, and Participant 3 experienced no change (mean increase $=2.6)$. 
At 1-month follow-up, ratings on the Conflict subscale of the FES were the same or better for Participants 1, 3, 4, and 5 with a mean change of -5.2 compared to post-treatment. For Cohesion, mothers of Participants 4 and 5 reported no change from post-treatment where as Participants 1, 2, and 3 indicated that they experienced slight decreases in family cohesion (mean change $=-5.2)$. Lastly, 1-month follow-up ratings on the Expressiveness subscale show that gains were maintained or improved more for Participants 1, 3, and 4, but Participants 2 and 5 had slight decreases in family expressiveness (mean change $=0$ ).

Problem Solving. As mentioned previously, the problem solving task was rated by mothers, youth, and an independent rater (see Table 7). For each of these ratings, higher scores indicated better problem solving skills. Over the course of treatment, participants and their mothers generally reported improvements in their use of problem solving skills. It is notable that the majority of participants and their mothers reported their highest ratings in week 8 which typically corresponded with the time this skill was practiced most often in sessions. At posttreatment, mothers of all five participants reported higher problem solving scores. In addition, four participants reported improvements while Participant 2 indicated no change from pretreatment. At 1-month follow-up only Participant 5's mother and child reports indicated additional improvements. All others reported lower scores for their participant in the problem solving task.

While participants and their mothers generally indicated improvements, the independent rater reported more variability in problem solving skills based on behavioral observations. Participants 1 and 5 were rated higher at post-treatment compared to pre-treatment, Participants 2 and 4 remained relatively stable (one point difference), and the interaction between Participant 3 and her mother resulted in a lower score at post-treatment. It is also notable, that the majority of 
participants received their highest score in week 8 which coincided with the time this skill was practiced the most in sessions. However, 1-month follow-up scores tended to be lower than post-treatment scores suggesting that treatment gains in this domain were not durable.

Global Response to Treatment. Figures 3 and 4 present clinician ratings based on the CGI. Clinician rated severity ratings (indicating the extent of severity and impairment associated with a given disorder) assigned to the principal diagnoses improved for $100 \%$ of the participants at post-treatment and 1-month follow-up. At pre-treatment, Participants 2, 3, and 5 were rated as markedly impaired (score of 5) while Participants 1 and 4 were rated as moderately impaired (score of 4$)($ mean rating $=4.6)$. At post-treatment, these ratings improved such that Participants 1 and 4 were rated as minimally impaired (score of 2), Participant 5 was rated as mildly impaired (score of 3), and Participants 2 and 3 were rated as moderately impaired (score of 4) (mean score = 3). At 1-month follow-up, these results were consistent with post-treatment ratings for four participants, but Participant 1 showed a slight increase in impairment as she moved from minimally impaired (score of 2) to moderately impaired (score of 3).

Clinician ratings of Global Improvement reflected these changes. At post-treatment, Participants 1 and 5 received a score of 2 indicating that they were much improved and Participants 2, 3, and 4 received a score of 3 indicating that they were minimally improved. At 1 month follow-up, these scores were generally the same although Participant 2 shifted from a rating of minimally improved (3) to a rating of much improved (2) to reflect his standing as "Student of the Month".

Ratings of improvement were also obtained from participants and their mothers (see Table 8). In general, ratings of improvement were reported to increase over the course of treatment as rated at weeks 5 and 8 as well as at post-treatment and 1-month follow-up. At post- 
treatment, mothers of Participants 1 and 5 rated their child as "slightly improved" (rating of 4) and mothers of Participants 2, 3, and 4 rated their child as "improved" (rating of 5). A mean rating of 4.6 at post-treatment indicated that on average the sample was "improved." Child ratings of improvement at post-treatment suggest that Participant 2 was "slightly improved" (rating of 4) and the other four indicated that they had "improved" (rating of 5). At 1-month follow-up, these ratings were somewhat lower suggesting that changes may not have been maintained. Participant 1's mother rated her as "slightly worse" (rating of 2), the mother of Participant 5 indicated "no change" (rating of 3), Participant 3's mother rated her daughter as "slightly improved" (rating of 4), and maternal ratings for Participants 2 and 4 suggested they had "improved" (rating of 5). On average, maternal ratings suggest that the sample was "slightly improved" (mean $=3.8$ ). Child ratings were slightly higher as Participants 1,2 , and 5 were "slightly improved" " (rating of 4) and Participants 3 and 4 were "improved" " (rating of 5). On average, participants indicated that in response to treatment they experienced "slight improvement" $($ mean $=4.4)$ at 1 -month follow-up.

Exploratory Analyses. As mentioned previously, paired t-tests were conducted in order to determine significant differences from pre-treatment to post-treatment. The majority of scores on the CBCL, YSR, and TRF showed no significant differences between pre-treatment and posttreatment. However, a significant decrease in symptoms was observed from pre-treatment to post-treatment on the Anxiety/Depression scale $(\mathrm{t}=3.01, \mathrm{p}<0.05)$. On the DBDRS, mothers' ratings of ODD symptoms showed significant improvement $(\mathrm{t}=4.951, \mathrm{p}<.01)$. In addition, clinician rated Severity of Illness showed significant improvements $(\mathrm{t}=6.53, \mathrm{p}<0.01)$. Significant differences were also evident for Emotion Regulation $(\mathrm{t}=-3.317, \mathrm{p}<.05)$, Family Cohesion $(\mathrm{t}=-3.135, \mathrm{p}<.05)$, maternal ratings of problem solving $(\mathrm{t}=-6.725, \mathrm{p}<.01)$, and child 
ratings of problem solving $(t=-3.516, \mathrm{p}<.05)$. Each of these suggests positive changes from pretreatment to post-treatment. However, other analyses showed no significant differences.

Composite Measures of Change. To assess treatment responder status, a composite criterion comprised of three measures was used (Jacobson \& Truax, 1991). A participant was considered a treatment responder if the following criteria were met: 1) a $30 \%$ improvement from pre- to post- treatment on mother-endorsed symptoms of ODD on the DBDRS, 2) a $30 \%$ improvement from pre- to post- treatment on child-endorsed symptoms of depression on the $\mathrm{CDI}$, and 3) rating of $1-2$ on the CGI. Based on these comprehensive criteria, only one participant (Participant 1) was considered a treatment responder.

In addition, the absolute level of functioning following treatment was determined by end state functioning. A participant was judged to have high end-state functioning if the following criteria applied: 1) mother ratings of ODD symptoms on the DBDRS in the non-clinical range (less than 4 symptoms rated as a 2 or 3; see Barkley, 1997), 2) child ratings of depressive symptoms on the CDI in the non-clinical range, 3) CGI rating of 1 (very much improved) or 2 (much improved), and 4) no diagnosis of ODD, CD, Major Depressive Disorder, or Dysthymia on the mother or child DISC. Only one participant (Participant 4) met these exacting criteria.

\section{Discussion}

The primary goal of this study was to develop and evaluate an integrated CBT intervention designed to simultaneously treat conduct problems and depression. The present results are promising as they provide tentative support for the treatment protocol. Specifically, all participants were retained in the treatment protocol and the majority of youth evidenced decreases in symptoms of either conduct problems or depression or both as well as improvements in diagnostic status. In addition, changes were evidenced in the family 
environment, emotion regulation, and problem solving skills in these comorbid youth. Thus, in support of the hypotheses, youth benefitted from treatment that specifically targeted symptoms of both conduct problems and depression and achieved somewhat better outcomes for both disorders.

Use of the multiple baseline design suggests that the treatment was effective in reducing symptoms of oppositionality for three of the five participants (Participants 1, 4, 5 according to parent report or Participants 1, 3, 4 according to youth self-report). It was also effective in decreasing symptoms of depression for four of the five participants according to mothers (Participants 1, 3, 4, and 5) or three participants according to youth self-report (Participants 1, 3, and 4). Although the follow-up period was brief, the findings indicate that treatment effects were durable for symptoms of depression, but less stable for symptoms of conduct problems. In addition, clinician ratings indicated global improvement for all participants with two participants identified as "Much Improved" at post-treatment and three identified as "Much Improved" at 1month follow-up. Participants and their mothers also endorsed global improvements as all participants were either "Improved" or "Slightly Improved" at post-treatment. However, participants and their mothers gave somewhat lower ratings of improvements at 1-month followup.

Changes in diagnostic status were also observed for each of the participants. In a comparison of pre- to post-treatment diagnoses, four participants (Participants 1, 2, 4, and 5) experienced positive change in conduct problem diagnoses (ODD or CD) and four participants (Participants 1, 2, 3, and 5) experienced positive change in diagnoses of depressive disorders (MDD or dysthymia). While only one participant was "diagnosis free" for both conduct problems and depression, others improved from a full diagnosis to a subclinical diagnosis or they 
met criteria for one of the conduct problem diagnoses (ODD or $\mathrm{CD}$ ) or one of the depression diagnoses (MDD or dysthymia) but not both.

As hypothesized, the current findings also demonstrate that the majority of families experienced less conflict, more cohesion, and greater expressiveness in the family environment. As mentioned previously, those with conduct problems and depression tend to have family environments marked by problems in these areas (George et al., 2006). As symptoms of these disorders were alleviated over the course of treatment, improvements were also evidenced in the family environment.

While the small sample in the present study prohibited analyses of moderation and mediation, some initial patterns emerged and suggest directions for future research. Although preliminary, it is interesting to compare demographic differences between those who were identified by the composite measures of change as being treatment responders (Participant 1) or having high end state functioning (Participant 4), to those who did not meet these criteria. It is notable that both of these participants were female, post-pubertal, and among the older children in the sample (above age 12). In addition, no parental psychopathology was reported at pretreatment for either of these participants. By contrast, Participant 2's self- and mother-reported symptoms indicated little change in response to treatment. This participant was pre-pubescent, male, one of the youngest participants, and his mother reported that she experienced high levels of anxiety. Thus, future studies should evaluate these factors as possible predictors of treatment outcome.

In terms of possible mechanisms of change, treatment in the present study included: (a) psychoeducation, (b) mood monitoring, (c) goal setting, (d) individual and family reinforcing activities, (e) affect identification and regulation, (f) individual and family problem solving, (g) 
cognitive restructuring, (h) communication, (i) social skills training, and (j) relapse prevention. Any one of these components, either alone or in combination, might have been responsible for the changes observed. However, only measures of emotion regulation and problem solving were included in the present study.

From pre- to post-treatment, four of the five participants showed improvements in Emotion Regulation and Lability/Negativity. Moreover, statistical analyses showed that this change was significant. Similarly, participants and their mothers reported improvements in their use of problem solving skills over the course of treatment. At post-treatment, mothers of all five participants reported higher problem solving scores. In addition, four of the five participants reported improvements. Statistical analyses showed that both mother and youth ratings of problem solving skills demonstrated significant improvements from pre- to post-treatment. However, behavioral observations of the problem solving task did not affirm significant improvement.

Evaluating patterns of symptomatic change occurring over the course of treatment may also reveal treatment specific effects, thereby pointing toward mechanisms of action. It is notable that participants and their mothers often reported their largest decrements in symptoms of both disorders between weeks 5 and 8 of treatment (see Participants 1, 3, and 5) which corresponded with the time problem solving skills were practiced most often in sessions. In addition, Participants 1, 4, and 5 received their highest rating from the independent rater who observed the problem solving task during week 8 . Thus, this pattern of findings demonstrates temporal precedence of the change in the proposed mediator (see Kazdin \& Nock, 2003). Taken together, the findings that children were better able to regulate emotion, express less negativity, and show improved problem solving skills (according to parent and youth report) are noteworthy given that 
the enhancement of these skills may be a first step in improving symptoms of conduct problems and depression.

It is helpful to compare the current findings to those of Rohde and colleagues (2005) who used the CWD-A treatment to treat youth diagnosed with conduct problems and depression. Following 16 2-hour sessions in the CWD-A course, Rohde et al. (2005) showed that at posttreatment $39 \%$ of youth no longer met criteria for depression and $9 \%$ no longer met criteria for CD. By comparison, in the current study, the one child who met full criteria for depression at pre-treatment did not meet diagnostic criteria at post-treatment and three of the four children with subclinical depression or dysthymia also experienced improvements. With regards to CD, all three youth who met criteria for $\mathrm{CD}$ at pre-treatment no longer met diagnostic criteria for this disorder at post-treatment. While all youth in the Rohde et al. (2005) study met full criteria for both depression and $\mathrm{CD}$, it is notable that mean dimensional symptoms of externalizing symptoms were higher in the current study than those reported by Rohde et al. (2005). Taken together, these findings suggest that the greater specificity in the current study may be associated with more diagnostic improvements in both conduct problems and depression than previous treatments with less specificity.

Given that treatment was relatively brief, it may be that the full effects of treatment were not yet evident for some participants. In the TADS study, clinician ratings indicated that it took 12 weeks for $50 \%$ of patients to achieve a stable response (rating of 1 or 2 on the CGI for 2 consecutive weeks) to CBT (Kratochwil et al., 2006). Because the current treatment provided only 11 sessions of treatment, on average, it is likely that treatment was not long enough to allow for higher rates of treatment response. This may be especially true because the current study sought to treat two disorders over this brief time period whereas TADS only targeted 
depression and included an acute phase as well as a maintenance phase. Still, if the TADS criteria were applied to the current study, only Participant 3, who received a CGI rating of 1 in weeks 9 and 10, achieved a stable response. If post-treatment ratings were substituted for week 12 ratings, Participant 5 also would have reached a stable response at week 12. Thus, results of the current study would show that $40 \%$ of these comorbid participants achieved a stable response by week 12 , approximating the $50 \%$ of participants in the TADS study who achieved a stable response. Still, it is probable that participants would have benefited from longer treatment duration (in both an acute and maintenance phase) as implemented in the TADS trial.

Although the majority of youth in the present study experienced improvements, the degree of change was minimal on several measures. In fact, exploratory analyses indicated that there were no significant differences in weekly symptom ratings from baseline to post-treatment on some measures, but there were significant reductions in symptoms of ODD (on the DBDRS) and anxiety or depression (on the CBCL) according to maternal reports. In addition, findings show significant improvements in the family environment, emotion regulation, and problem solving skills (as rated by the parent and child). Although a limited number of significant differences were found, these findings are notable given the small sample size and limited power.

In part, the minimal levels of change may have been due to initial symptom ratings being below clinical cutoff scores; as a result, it was difficult for participants to demonstrate significant improvement. This is particularly true for the composite measure of change that required a $30 \%$ improvement from pre-to post-treatment. Thus, only one participant was classified as a treatment responder and one participant met criteria for high end state functioning. Typically, a participant with high end state functioning would be a treatment responder, but because this participant was near the normative range at pre-treatment, she was not classified as a treatment responder. 
Moreover, the lack of consistency of findings across measures using multiple informants further suggests that these findings may not be reliable. Although such discrepancies are not uncommon in the literature (for a recent review, see De Los Reyes \& Kazdin, 2005), they present challenges to the interpretation of findings. Typically, children and adolescents in clinically referred samples tend to report fewer externalizing and more internalizing symptoms than their parents (Smith, 2007). However, in the current study, mothers tended to rate both internalizing and externalizing problems higher than their son or daughter. Similar disagreement was also observed on the DISC as symptoms were endorsed by parents but not adolescents. Various factors may influence these ratings. For example, considerable research demonstrates the impact of maternal psychopathology, family conflict, and social desirability on ratings of youth behavior (Bingham, Loukas, Fitzgerald, \& Zucker, 2003; Merydith, Prout, \& Blaha, 2003). In the present study, self-reports suggesting that both internalizing and externalizing behaviors were low may be related to the oppositionality of these youth and their unwillingness to endorse either type of disorder. Alternatively, it may be that mothers over-reported symptoms.

The current study also made use of teacher ratings of internalizing and externalizing symptoms. On average, these ratings suggested minimal improvements in symptoms of depression whereas they indicated that symptoms of externalizing disorders failed to improve at post-treatment compared to pre-treatment. While these symptoms may be an accurate representation of the youth's problems in those settings, these finding may also relate to the timing of the intervention. All participants began treatment in the first half of the academic year and it may be that teachers were less familiar with some participants' symptoms at that time. Thus, differences in their pre-treatment versus post-treatment ratings may partially reflect their level of familiarity with these participants. 
In addition, the current study included behavioral observation to assess parent-adolescent interactions during a problem solving task. These ratings suggested that problem solving skills varied over the course of treatment and that there were some discrepancies with participant (mother and youth) ratings compared to behavioral observations. Again, there may be several explanations for this. First, participants and their mothers may have wanted to present themselves in a more positive manner. Second, this discrepancy may relate to differences in the content of the behavior rating versus the parent and adolescent self-reported questionnaires about the task. Specifically, the behavioral observation pertained only to the problem solving task while the problem solving questionnaire completed by participants and their mothers also contained a question about their use of problem solving skills at home. The behavioral rating also evaluated more of the problem solving process while the questionnaires asked more specifically about the outcomes of the task.

A related measurement problem is the considerable week-to-week variations in symptom ratings during baseline periods and treatment phases that attest to the unstable nature of these symptoms as well as their potential reactivity to recordings. It is notable that both youth and their mothers tended to have strong reactions to seemingly minor occurrences over the course of treatment that were reflected in their weekly ratings. While some major events occurred (e.g., cutting, running away), other minor events (e.g., not going to bed on time, not doing chores) were more common and ratings sometimes reflected the reactivity of mothers and youth to these seemingly minor events. In general, clinician ratings tended to be more stable and may have provided a more objective rating. However, even the clinician ratings showed some variability from week to week suggesting that symptoms did not decrease in a linear pattern and treatment implementation did not result in immediate or persistent reductions in symptoms. It is also 
possible that some of this variability is related to the extinction burst that is sometimes observed in the treatment of children with behavior problems (Lerman \& Iwata, 1995). This phenomenon involves an initial increase in the frequency or severity of problem behaviors in response to intervention as the child "tests the limits."

In addition, the lack of changes at the beginning of treatment or worsening of symptoms in some participants may be related to the fact that the first sessions are devoted primarily to psychoeducation and mood/conflict monitoring and parents and their children do not learn or practice skills until later in treatment. In fact, it is possible that by beginning to monitor symptoms at the beginning of treatment, mothers and their sons or daughters became more aware of the frequency of behaviors, and thus their ratings at the beginning of treatment were sometimes higher than baseline scores. This is not an uncommon phenomenon in psychotherapy research and practice.

Because an independent evaluator was not used in the present study, it is difficult to determine the accuracy of reports provided by multiple informants. Parent, teacher, self, and clinician reports each provide valuable information in assessing how the adolescent is behaving across settings, however, it is possible that these ratings were influenced by various biases (e.g., experimenter bias). An independent rater, blind to treatment status, would have provided a more accurate assessment of the adolescents' functioning over the course of treatment. Thus, future studies should use an independent evaluator.

\section{Strengths and Limitations}

The primary strength of the current study was the use of a nonconcurrent multiple baseline design which measured changes in symptoms of conduct problems and depression in individual participants from week to week. This design allowed for a greater understanding of 
the treatment than group designs since it provided more detail on individual responses to treatment from week to week. Such a design was especially helpful given that this is a preliminary investigation of the treatment protocol. It should also be noted that the multiple baseline design controls for several internal validity threats including maturation and exposure (Carr, 2005). Additional strengths include the use of multiple informants, a diagnostic interview, a standardized treatment protocol, treatment adherence and competency ratings, 1-month followup assessments, and inclusion of behavior observations (i.e., problem solving task).

However, the current study is not without limitations. Most importantly, while there are several benefits to using a single subject design, there are also disadvantages. In particular, use of a multiple-baseline approach limited the ability to conduct statistical analyses. Thus, conclusions about the data are based primarily on visual inspection and are subject to (mis)interpretation. In addition, the study did not include a comparison condition other than the staggered baseline. Future studies may consider comparing the current treatment protocol to an empirically supported treatment for either conduct problems or depression. Lastly, a primary limitation of the non-concurrent multiple baseline design is the inability to identify history effects that might coincide with treatment implementation (Gliner et al., 2000). For example, the current study may have been affected by the implementation of treatment early in the academic year or events related to the holiday season (i.e., spending more time with family, time off from treatment during this time).

In addition, the fact that symptom improvement sometimes occurred before treatment was implemented (see Figures 1 and 2) and the lack of stability in baseline ratings call the validity of some of the findings into question. As reviewed by Christ (2007), problems with either trend or variability can be resolved more often within a concurrent multiple baseline 
design than with a non-concurrent multiple baseline design, as researches can extend the baseline phase until the baseline pattern stabilizes. In contrast, the non-concurrent multiple baseline design requires that the baseline duration be specified and assigned a priori, prohibiting its extension and increasing the probability that problematic baseline scores may threaten the validity of findings (Watson \& Workman, 1981). When excessive variability and downward trends occur, it is recommended that such participants be excluded from the study. However, given the exploratory nature of the present study, such participants were not excluded. Thus, while outcomes for these participants are reported, they should be interpreted with appropriate caution.

Along these lines, it should also be noted that the majority of findings relied on differences between pre- and post-treatment ratings rather than the week to week changes that are typically reported in multiple baseline studies. Measures administered on a weekly basis failed to show consistent change although overall differences were observed from pre- to posttreatment. The weekly ratings of depression and conduct problems tended to reflect participants current "state" whereas pre- and post-treatment ratings pertain to their "trait." Thus, although they frequently experienced oppositional or depressive episodes, their overall level of symptoms decreased from pre- to post-treatment.

Finally, several other possible limitations are worth mentioning. As mentioned previously, there were issues related to the non-clinical symptom ratings of some participants at pre-treatment. Similarly, only one participant met full criteria for depression at pre-treatment according to the diagnostic interview. These low scores and subclinical depression diagnoses substantially limited the possibility of clinical improvements. Moreover, the pre-treatment nonclinical ratings made it difficult for participants to achieve the status of "treatment responder" or 
"high end state functioning." Second, sampling biases may have limited the generalizability of the findings. Many families were self-referred, well educated, and two-parent households. Moreover, because these youth were near the normative range on some measures and four of the five participants did not meet full criteria for depression at pre-treatment, it is not known how effective the treatment would be for those with more severe symptoms of conduct problems and depression. Thus, these findings may not generalize to those with different presentations or those with more severe afflictions. Third, although medication was stable throughout treatment, it cannot be determined whether medication contributed to any improvement. Lastly, the followup assessment occurred 1-month after the completion of treatment and future studies should assess the maintenance of gains over a longer period. At later follow up points it is possible that further improvements would be made as these skills are further practiced, or it may be that the skills trained are not used and a loss of gains may be observed. Along these lines, it is likely that participants would benefit from a maintenance phase of treatment that would allow for further practice of the skills trained and a greater chance that skills would be maintained at follow-up points.

\section{Clinical Implications}

Despite these limitations, this study is the first to evaluate the effectiveness of an integrated treatment for children with co-occurring conduct problems and depression. While it is premature to derive major clinical implications from a pilot study, the treatment evaluation process did provide preliminary evidence in support of the integrated CBT protocol for youth with conduct problems and depression. Thus, combining existing treatments to address comorbid problems in a simultaneous fashion could hold promise for enhancing treatment effectiveness. These findings extend child psychotherapy research in an important direction. Given the lack of 
evidence-based methods available to researchers and clinicians for simultaneously treating comorbid disorders, the intervention developed and evaluated in this study appears promising. Integrated CBT treatment protocols for depression and conduct problems have potential for improving current treatments by simultaneously treating symptoms of both disorders. Future research will need to systematically examine this approach in a randomized clinical trial and determine the transportability of the approach to real-life clinical settings. 


\section{References}

Achenbach, T.M. (2001a). Manual for the Child Behavior Checklist 4-18 and 2001 profile. Burlington, VT: Department of Psychiatry, University of Vermont.

Achenbach, T.M. (2001b). Manual for the Teacher's Report Form and 2001 profile. Burlington, VT: Department of Psychiatry, University of Vermont.

Achenbach, T.M. (2001c). Manual for the Youth Self Report and 2001 profile. Burlington, VT: Department of Psychiatry, University of Vermont.

American Psychiatric Association. (2000). The diagnostic and statistical manual of mental disorders ( $4^{\text {th }}$ ed., text revised). Washington, DC: Author.

Angold, A., Costello, E. J., \& Erkanli, A. (1999). Comorbidity. Journal of Child Psychology and Psychiatry, 40, 57-87.

Arredondo, D.E. \& Butler, S.F. (1994). Affective comorbidity in psychiatrically hospitalized adolescents with conduct disorder or oppositional defiant disorder: Should conduct disorder be treated with mood stabilizers? Journal of Child and Adolescent Psychopharmacology, 4(3), 151-158.

Bandura, A. (1986). Social foundations of thought and action: A social cognitive theory. Prentice Hall: Saddle River, NJ.

Barkely, R.A. (1997). Defiant children: A clinician's manual for parent training, $2^{\text {nd }}$ Edition. New York: Guilford.

Beauchaine, T. P., Webster-Stratton, C., \& Reid, M. J. (2005). Mediators, moderators, and predictors of one-year outcomes among children treated for early-onset conduct problems: A latent growth curve analysis. Journal of Consulting and Clinical Psychology, 73, 371-388. 
Beck, A.T., Rush, A.J., Shaw, B.F., \& Emery, G. (1979). Cognitive Therapy of Depression. Guilford: New York.

Bennett, D. S., \& Gibbons, T. A. (2000). Efficacy of child cognitive behavioral interventions for antisocial behavior: A meta-analysis. Child and Family Behavior Therapy, 22, 1-15.

Biederman, J., Faraone, S., Mick, E., \& Lelon, E. (1995). Psychiatric comorbidity among referred juveniles with major depression: Fact or artifact? Journal of the American Academy of Child and Adolescent Psychiatry, 34(5), 579-590.

Brestan, E. V. \& Eyberg, S. M. (1998). Effective psychosocial treatment of conduct-disordered children and adolescents: 29 years, 82 studies, and 5,272 kids. Journal of Clinical Child Psychology, 27, 180-189.

Capaldi, D. \& Stoolmiller, M. (1999). Co-occurrence of conduct problems and depressive symptoms in early adolescent boys: III. Prediction to young-adult adjustment. Development and Psychopathology, 11(1), 59-84.

Capaldi, D. (1991). Co-occurrence of conduct problems and depressive symptoms in early adolescent boys: I. Familial factors and general adjustment at Grade 6. Development and Psychopathology, 3(3), 277-300.

Capaldi, D. (1992). Co-occurrence of conduct problems and depressive symptoms in early adolescent boys: II. A 2-year follow-up at Grade 8. Development and Psychopathology, $4(1), 125-144$.

Carr, J. (2005). Recommendations for reporting multiple-baseline designs across participants. Behavioral Interventions, 20(3), 219-224.

Carver, C.S. \& Scheier, M.F. (1996). Perspectives on personality. Allyn \& Bacon: Needham Heights, MA. 
Chorpita, B. F., Yim, L. M., Donkervoet, J. C., Arensdorf, A., Amundsen, M. J., McGee, C., et al. (2002). Toward large-scale implementation of empirically supported treatments for children: A review and observations by the Hawaii empirical basis to services task force. Clinical Psychology: Science and Practice, 9, 165-190.

Cohen, P., Cohen, J., Kasen, S., Velez, C. N., Hartmark, C., Johnson, J., et al.(1993). An epidemiological study of disorders in late childhood and adolescence: I. Age- and genderspecific prevalence. Journal of Child Psychology \& Psychiatry, 34, 851-867

Costin, J. \& Chambers, S. (2007). Parent management training as a treatment for children with oppositional defiant disorder referred to a mental health clinic. Clinical Child Psychology and Psychiatry, 12, 511-524.

Crick, N. R. \& Dodge, K. A. (1994). A review and reformulation of social information processing mechanisms in children's social adjustment. Psychological Bulletin, 115, 74101.

Curry, J.F. (2001). Specific psychotherapies for childhood and adolescent depression. Biological Psychiatry, 49(12), 1091-1100.

Curry, J.F., Wells, K.C., \& Lochman, J.E. (2003). Cognitive-behavioral intervention for depressed, substance-abusing adolescents: Development and pilot testing. Journal of the American Academy of Child \& Adolescent Psychiatry, 42(6), 656-665.

Derogatis. L. R. (1994). SCL-90-R: Administration, scoring, and procedures manual. Available from author.

Dishion, T. J. \& Patterson, G. R. (1992). Age effects in parent training outcomes. Behavior Therapy, 23, 719-729. 
Dodge, K.A. (1993). Social-cognitive mechanisms in the development of conduct disorder and depression. Annual Review of Psychology, 44, 559-584.

Durlak, J. A., Fuhrman, T. \& Lampman, C. (1991). Effectiveness of cognitive-behavior therapy for maladapting children: A meta-analysis. Psychological Bulletin, 110, 204-214.

Ezpeleta, L., Domènech, J., \& Angold, A. (2006). A comparison of pure and comorbid CD/ODD and depression. Journal of Child Psychology and Psychiatry, 47(7), 704-712.

Fergusson, D.M., Lynskey, M.T., \& Horwood, L.J. (1996). Origins of comorbidity between conduct and affective disorders. Journal of the American Academy of Child and Adolescent Psychiatry, 35 (4), 451-460.

Fleming, J.E., Boyle, M.H., \& Offord, D.R. (1993). The outcome of adolescent depression in the Ontario Child Health Study follow-up. Journal of the American Academy of Child and Adolescent Psychiatry, 32, 28-33.

Frick, P. J. (1998). Conduct disorders. In: Handbook of child psychopathology (3rd ed.). T.H. Ollendick \& M. Hersen (Eds.) New York: Plenum Press, 213-237.

Frick, P. J. (2001). Effective interventions for children and adolescents with conduct disorder. Canadian Journal of Psychiatry, 46(7), 597-608.

Frick, P. J., Lahey, B. J., Applegate, B., Kerdyck, L., Ollendick, T. H., Hynd, G. W., Garfinkel, B., Greenhill, L., Biederman, J., Barkley, R. A., McBurnett, K., Newcorn, J., \& Waldman, I. (1994). DSM-IV field trials for the disruptive behavior disorders: Symptom utility estimates. Journal of the American Academy of Child and Adolescent Psychiatry, $33,529-539$ 
Friman, P.C. Handwerk, M.L., \& Smith, G.L. (2000). External validity of conduct and oppositional defiant disorders determined by the NIMH Diagnostic Interview Schedule for Children. Journal of Abnormal Child Psychology, 28(3), 277-286.

Greene, R. W. (1998). The explosive child: Understanding and parenting easily frustrated, “chronically inflexible” children. New York: HarperCollins.

Greene, R.W., Biederman, J., Zerwas, S., Monuteaux, M., Goring, J.C., \& Faraone, S.V. (2002). Psychiatric comorbidity, family dysfunction, and social impairment in referred youth with oppositional defiant disorder. American Journal of Psychiatry, 159(7), 1214-1224.

Guy, W. (1976). The clinical global impression scale. As published in The ECDEU Assessment Manual for Psychopharmacology- Revised (DHEW Publ No ADM 76-338). Rockville, MD, U.S. Department of Health, Education, and Welfare Public Health Service, Alcohol, Drug Abuse,Mental Health Administration, NIMH Psychopharmacology Research Branch, Division of Extramural Research, p. 218-222.

Harrington, R., Fudge, H., Rutter, M., Pickles, A. (1991). Adult outcomes of childhood and adolescent depression: II. Links with antisocial disorders. Journal of the American Academy of Child and Adolescent Psychiatry, 30(3), 434-439.

Hughes, C., Preskorn, S.H., Weller, E., Weller, R., Hassanein, R., \& Tucker, S. (1990). The effect of concomitant disorders in childhood depression on predicting treatment response. Psychopharmacology Bulletin. 26(2), 235-238.

Jacobson, N. S. \& Truax, P. (1991). Clinical significance: A statistical approach to defining meaningful change in psychotherapy research. Journal of Consulting and Clinical Psychology, 59, 12-19. 
Johnston, J.M. \& Pennypacker, H.S. (1993). Strategies and tactics of behavioral research $\left(2^{\text {nd }}\right.$ ed.). Hillsdale, NJ, England: Lawrence Erlbaum Associates, Inc.

Kaslow, N.J. \& Thompson, M.P. (1998). Applying the criteria for empirically supported treatments to studies of psychosocial interventions for child and adolescent depression. Journal of Clinical Child Psychology, 27(2), 146-155.

Kazdin, A. E. (1995). Child, parent, and family dysfunction as predictors of outcome in cognitive-behavioral treatment of antisocial children. Behaviour Research and Therapy, $33,271-281$.

Kazdin, A. E., Esveldt-Dawson, K., French, N.H., \& Unis, A.S. (1987a). Effects of parent management training and problem-solving skills training combined in the treatment of antisocial child behavior. Journal of the American Academy of Child and Adolescent Psychiatry, 26, 416-424.

Kazdin, A. E., Esveldt-Dawson, K., French, N.H., \& Unis, A.S. (1987b). Problem solving skills training and relationship therapy in the treatment of antisocial child behavior. Journal of Consulting and Clinical Psychology, 55, 76-85.

Kazdin, A. E., Sigel, T.C., \& Bass, D. (1992). Cognitive problem-solving skills training and parent management training in the treatment of antisocial behavior in children. Journal of Consulting and Clinical Psychology, 60, 733-747.

Kazdin, A.E. \& Weisz, J.R. (1998). Identifying and developing empirically supported child and adolescent treatments. Journal of Consulting and Clinical Psychology, 66(1), 19-36.

Kazdin, A.E. (2005). Parent management training: Treatment for oppositional, aggressive, and antisocial behavior in children and adolescents. New York: Oxford University Press. 
Keiley, M., Lofthouse, N., Bates, J.E., Dodge, K.A., \& Pettit, G.S. (2003). Differential risks of covarying and pure components in mother and teacher reports of externalizing and internalizing behavior across ages 5 to 14. Journal of Abnormal Child Psychology, 31(3), 267-283.

Kovacs, M. (1992). Children's Depression Inventory manual. North Tonawanda, NY: MultiHealth Systems.

Kovacs, M., Paulauskas, S., Gatsonis, C., \& Richards, C. (1988). Depressive disorders in childhood: III. A longitudinal study of comorbidity with and risk for conduct disorders. Journal of Affective Disorders, 15(3), 205-217.

Kovacs, M., Paulauskas, S., Gatsonis, C., \& Richards, C. (1988). Depressive disorders in childhood: III. A longitudinal study of comorbidity with and risk for conduct disorders. Journal of Affective Disorders, 15(3), 205-217.

Lahey, B. B., Applegate, B., Barkley, R. A., Garfinkel, B., McBurnett, K., Kerdyke, L., Greenhill, L., Hund, G. W., Frick, P. J, Newcorn, J., Biederman, J., Ollendick, T. H., Hart, E., Perez, D., Waldman, I., \& Shaffer, D. (1994). DSM-IV field trials for Oppositional Defiant Disorder and Conduct Disorder in children and adolescents. American Journal of Psychiatry, 151, 1163-1171.

Lahey, B.B., Loeber, R., Burke, J., Rathouz, P.J., \& McBurnett, K. (2002). Waxing and waning in concert: Dynamic comorbidity of conduct disorder with other disruptive and emotional problems over 17 years among clinic-referred boys. Journal of Abnormal Psychology, $111(4), 556-567$. 
Lewinsohn, P. M., Hops, H., Roberts, R. E., Seeley, J. R., \& Andrews, J. A. (1993). Adolescent psychopathology: 1. Prevalence and incidence of depression and other DSM-III-R disorders in high school students. Journal of Abnormal Psychology, 102, 133-144.

Lewinsohn, P. M., Rohde, P., \& Seeley, J.R. (1995). Adolescent psychopathology: III. The clinical consequences of comorbidity. Journal of the American Academy of Child and Adolescent Psychiatry, 34(4), 510-519.

Lochman, J. \& Wells, K. (1996). A social-cognitive intervention with aggressive children: Prevention effects and contextual implementation issues. In R. D. Peters \& R. J. McMahon, Preventing childhood disorders, substance abuse, and delinquency, 111143. Thousand Oaks, CA, US: Sage Publications, Inc.

Loeber, R., \& Keenan, K. (1994). Interaction between conduct disorder and its comorbid conditions: Effects of age and gender. Clinical Psychology Review, 14(6), 497-523.

Loeber, R., Russo, M.F., Stouthamer-Loeber, M., \& Lahey, B.B. (1994). Internalizing problems and their relation to the development of disruptive behaviors in adolescence. Journal of Research on Adolescence, 44(4), 615-637.

McCart, M., Priester, P., Davies, W., \& Azen, R. (2006). Differential effectiveness of behavioral parent training and cognitive behavioral therapy for antisocial youth: A meta-analysis. Journal of Abnormal Child Psychology, 34, 527-543.

McMahon, R.J., \& Forehand, R.L. (2003). Helping the noncompliant child: Family-based treatment for oppositional behavior (2nd ed.). New York, NY, US: Guilford Press.

Moos, R.H. \& Moos, B.S. (1981). Manual for the Family Environment Scale. Palo Alto, California: Consulting Psychologists Press. 
Nottelmann, E. \& Jensen, P. (1997). Comorbidity of disorders in childhood and adolescence: Developmental Perspectives. In T.H. Ollendick \& R.J. Prinz (Eds.), Advances in Clinical Child Psychology, 18. New York: Plenum Press.

Ollendick, T. H. \& King, N. J. (2004). Empirically supported treatments for children: Advances toward evidence-based practice. In P. Barrett \& T. H. Ollendick (Eds.), Handbook of interventions that work with children and adolescents: From prevention to treatment (pp. 3-26). London: John Wiley \& Sons, Inc.

Patterson, G. R., Reid, J. B. \& Dishion, T. J. (1992). Antisocial boys. Eugene, OR: Castalia.

Pelham, W.E., Gnagy, E.M., \& Greenslade, K.E. (1992).Teacher ratings of DSM-III--R symptoms for the disruptive behavior disorders. Journal of the American Academy of Child and Adolescent Psychiatry, 31(2), 210-218.

Petersen, A. C., Crockett, L., Richards, M., \& Boxer, A. (1988). A self-report measure of pubertal status: Reliability, validity, and initial norms. Journal of Youth and Adolescence, 17, 117-133.

Piacentini, J.C., Cohen, P., \& Cohen, J. (1992). Combining discrepant diagnostic information from multiple sources: Are complex algorithms better than simple ones? Journal of Abnormal Child Psychology, 20(1), 51-63.

Puig-Antich, J. (1982). Major depression and conduct disorder in prepuberty. Journal of the American Academy of Child Psychiatry, 21(2), 118-128.

Rapp, L.H., Wodarski, J.S. (1997). The comorbidity ot conduct disorder and depression in adolescents: A comprehensive interpersonal treatment technology. Family Therapy, 24 (2), 81-100. 
Robins, L.N. \& Price, R. (1991). Adult disorders predicted by childhood conduct problems: Results from the NIMH Epidemiologic Catchment Area project. Psychiatry: Journal for the Study of Interpersonal Processes, 54(2), 116-132.

Rohde, P., Clarke, G.N., Lewinsohn, P.M., Seeley, J.R., \& Kaufman, N.K. (2001).Impact of comorbidity on a cognitive-behavioral group treatment for adolescent depression. Journal of the American Academy of Child and Adolescent Psychiatry, 40(7), 795-802.

Rohde, P., Clarke, G.N., Mace, D.E., Jorgensen, J.S., \& Seeley, J.R. (2004). An efficacy/effectiveness study of cognitive-behavioral treatment for adolescents with comorbid major depression and conduct disorder. Journal of the American Academy of Child and Adolescent Psychiatry, 43(6), 660-668.

Ruma, P. R., Burke, R. V. \& Thompson, R. W. (1996). Group parent training: Is it effective for children of all ages? Behavior Therapy, 27, 159-169.

Schwab-Stone, M.E., Shaffer, D., Dulcan, M.K. Jensen, P.S. Fisher, P., Bird, H.R., Goodman, S.H., Lahey, B.B., Lichtman, J. H., Canino, G., Rubio-Stipec, M., \& Rae, D.S. (1996).Criterion validity of the NIMH Diagnostic Interview Schedule for Children, Version 2.3 (DISC-2.3). Journal of the American Academy of Child and Adolescent Psychiatry, 35, 878-888.

Seligman, L. D., Goza, A. B., \& Ollendick, T. H. (2004). Treatment of depression in children and adolescents. In T. H. Ollendick \& P. Barrett (Eds.), Handbook of interventions that work with children and adolescents: From prevention to treatment (pp. 301-328). London: Wiley.

Serketich, W. J., \& Dumas, J. E. (1996). The effectiveness of behavioral parent training to modify antisocial behavior in children: A meta-analysis. Behavior Therapy, 27, 171-186. 
Shaffer, D., Fisher, P., \& Lucas, C.P. (2000). NIMH Diagnostic Interview Schedule for Children Version IV (NIMH DISC-IV): Description, differences from previous versions, and reliability of some common diagnoses. Journal of the American Academy of Child and Adolescent Psychiatry, 39(1), 28-38.

Shaffer, D., Fisher, P., Dulcan, M.K., Davies, M., Piacentini, J., Schwab-Stone, M.E., Lahey, B.B., Bourdon, K., Jensen, P., Bird, H.R., Canino, G., \& Regier, D.A. (1996). The NIMH Diagnostic Interview Schedule for Children, Version 2.3 (DISC-2.3): description, acceptability, prevalence rates, and performance in the MECA study. Journal of the American Academy of Child and Adolescent Psychiatry ,35:865-877.

Simic, M., \& Fombonne, E. (2001). Depressive conduct disorder: Symptom patterns and correlates in referred children and adolescents. Journal of Affective Disorders, 62, 175185.

Smucker, M.R., Craighead, W.E., \& Craighead, L.W. (1986). Normative and reliability data for the Children's Depression Inventory. Journal of Abnormal Child Psychology, 14(1), 2539.

Spivack, G., \& Shure, M. (1982). The cognition of social adjustment: Interpersonal cognitive problem-solving thinking. In B. B. Lahey \& A. E. Kazdin (Eds.), Advances in clinical child psychology (Vol. 5, pp. 323-369). New York: Plenum Press.

Treatment for Adolescents with Depression Study (TADS) Team (2004). Fluoxetine, cognitivebehavioral therapy, and their combination for adolescents with depression: Treatment for Adolescents with Depression Study (TADS) randomized controlled trial. Journal of the American Medical Association, 292(7), 807-820. 
Webster-Stratton, C., Reid, J., \& Hammond, M. (2001). Social skills and problem-solving training for children with early-onset conduct problems: Who benefits? Journal of Child Psychology and Psychiatry, 42, 943-952.

Weschler, D. (2003). Manual for the Wechsler Intelligence Scale for Children-Third Edition. San Antonio, TX: Psychological Corporation.

Wolff, J.C. \& Ollendick, T.H. (2006). The comorbidity of conduct problems and depression in childhood and adolescence. Clinical Child and Family Psychology Review, 9, 201-220.

Zoccolillo, M. \& Rogers, K. (1991). Characteristics and outcome of hospitalized adolescent girls with conduct disorder. Journal of the American Academy of Child and Adolescent Psychiatry, 30(6), 973-981. 
Table 1. Participant Characteristics

\begin{tabular}{|c|c|c|c|c|c|c|}
\hline Participant & Sex & Age & Race & $\begin{array}{c}\text { Pre-Treatment } \\
\text { Diagnosis }\end{array}$ & $\begin{array}{c}\text { Pubertal } \\
\text { Status }\end{array}$ & $\begin{array}{c}\text { Maternal } \\
\text { Psychopathology }\end{array}$ \\
\hline 1 & $\mathrm{~F}$ & 14 & $\begin{array}{c}\text { African } \\
\text { American }\end{array}$ & $\begin{array}{c}\text { ODD } \\
\text { CD } \\
\text { Subclinical MDD } \\
\text { Subclinical DYS } \\
\text { Mania } \\
\text { SOC }\end{array}$ & $\begin{array}{c}\text { Post- } \\
\text { pubertal }\end{array}$ & None \\
\hline 2 & $\mathrm{M}$ & 11 & Caucasian & $\begin{array}{c}\text { ODD } \\
\text { CD } \\
\text { Subclinical MDD } \\
\text { ADHD } \\
\text { SAD } \\
\text { Mania }\end{array}$ & $\begin{array}{c}\text { Pre- } \\
\text { pubertal }\end{array}$ & Anxiety \\
\hline 3 & $\mathrm{~F}$ & 11 & Caucasian & $\begin{array}{l}\text { ODD } \\
\text { MDD } \\
\text { SAD }\end{array}$ & $\begin{array}{l}\text { Post- } \\
\text { pubertal }\end{array}$ & $\begin{array}{c}\text { Paranoid Ideation } \\
\text { Depression } \\
\text { Interpersonal } \\
\text { Sensitivity }\end{array}$ \\
\hline 4 & $\mathrm{~F}$ & 13 & Caucasian & $\begin{array}{c}\text { CD } \\
\text { Subclinical MDD }\end{array}$ & $\begin{array}{c}\text { Post- } \\
\text { pubertal }\end{array}$ & None \\
\hline 5 & $\mathrm{M}$ & 14 & Caucasian & $\begin{array}{c}\text { ODD } \\
\text { CD } \\
\text { Subclinical MDD } \\
\text { Subclinical DYS } \\
\text { ADHD } \\
\text { SOC }\end{array}$ & $\begin{array}{l}\text { Post- } \\
\text { pubertal }\end{array}$ & None \\
\hline
\end{tabular}

$\mathrm{CD}=$ Conduct Disorder; ODD $=$ Oppositional Defiant Disorder; $\mathrm{MDD}=$ Major Depressive Disorder; DYS $=$ Dysthymia; $\mathrm{SOC}=$ Social Phobia $; \mathrm{SAD}=$ Separation Anxiety Disorder; $\mathrm{ADHD}=$ Attention Deficit-Hyperactivity Disorder 
Table 2. Mother and Child Weekly Symptom Ratings Based on the Conduct Problem and Depression Rating Scale

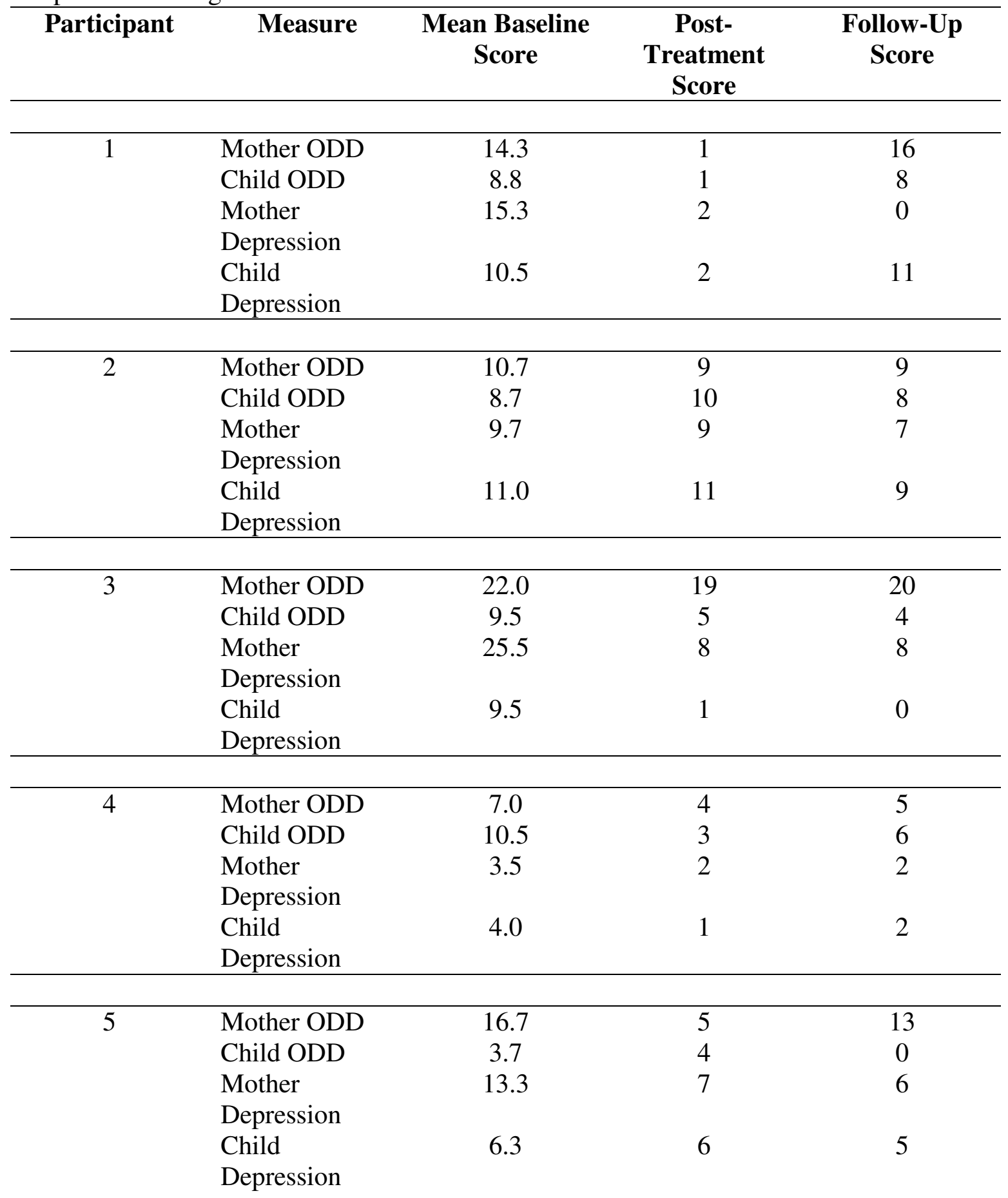


Table 3. Participant Diagnoses by Phase of Treatment

\begin{tabular}{|c|c|c|c|}
\hline Participant & $\begin{array}{c}\text { Pre-Treatment } \\
\text { Diagnosis }\end{array}$ & $\begin{array}{c}\text { Post-Treatment } \\
\text { Diagnosis }\end{array}$ & $\begin{array}{c}\text { 1-Month } \\
\text { Follow-Up }\end{array}$ \\
\hline 1 & $\begin{array}{c}\text { ODD } \\
\text { CD } \\
\text { Subclinical MDD } \\
\text { Subclinical DYS } \\
\text { Mania } \\
\text { SOC }\end{array}$ & $\begin{array}{c}\text { ODD } \\
\text { Subclinical MDD }\end{array}$ & ODD \\
\hline 2 & $\begin{array}{c}\text { ODD } \\
\text { CD } \\
\text { Subclinical MDD } \\
\text { ADHD } \\
\text { SAD } \\
\text { Mania }\end{array}$ & $\begin{array}{l}\text { ADHD } \\
\text { SAD } \\
\text { Mania }\end{array}$ & $\begin{array}{c}\text { Subclinical ODD } \\
\text { ADHD } \\
\text { SAD } \\
\text { Mania }\end{array}$ \\
\hline 3 & $\begin{array}{l}\text { ODD } \\
\text { MDD } \\
\text { SAD }\end{array}$ & $\begin{array}{c}\text { ODD } \\
\text { Subclinical DYS }\end{array}$ & $\begin{array}{c}\text { ODD } \\
\text { Subclinical DYS } \\
\text { Subclinical MDD }\end{array}$ \\
\hline 4 & $\begin{array}{c}\text { CD } \\
\text { Subclinical MDD }\end{array}$ & $\begin{array}{c}\text { ODD } \\
\text { Subclinical MDD }\end{array}$ & Subclinical ODD \\
\hline 5 & $\begin{array}{c}\text { ODD } \\
\text { CD } \\
\text { Subclinical MDD } \\
\text { Subclinical DYS } \\
\text { ADHD } \\
\text { SOC }\end{array}$ & $\begin{array}{l}\text { ODD } \\
\text { ADHD }\end{array}$ & $\begin{array}{c}\text { ODD } \\
\text { Subclinical Dysthymia }\end{array}$ \\
\hline
\end{tabular}

$\mathrm{CD}=$ Conduct Disorder; ODD = Oppositional Defiant Disorder; MDD = Major Depressive Disorder; DYS = Dysthymia; SOC = Social Phobia; SAD = Separation Anxiety Disorder; $\mathrm{ADHD}=$ Attention Deficit-Hyperactivity Disorder 
Table 4. Behavior Checklist T-Scores for Each Participant and Overall Means

\begin{tabular}{|c|c|c|c|c|c|}
\hline Participant & Rater & Measure & $\begin{array}{c}\text { Pre- } \\
\text { Treatment }\end{array}$ & $\begin{array}{c}\text { Post- } \\
\text { Treatment } \\
\end{array}$ & $\begin{array}{c}\text { Follow- } \\
\text { Up }\end{array}$ \\
\hline \multirow[t]{12}{*}{1} & \multirow[t]{4}{*}{ Mother } & Anxious/Depressed & $70 *$ & 65 & 59 \\
\hline & & Withdrawn/Depressed & 63 & 60 & 57 \\
\hline & & Rule-Breaking & 68 & 62 & 57 \\
\hline & & Aggressive & $75 *$ & $70 *$ & $70 *$ \\
\hline & \multirow[t]{4}{*}{ Teacher } & Anxious/Depressed & 54 & 68 & 68 \\
\hline & & Withdrawn/Depressed & 50 & 52 & 52 \\
\hline & & Rule-Breaking & 69 & 67 & 67 \\
\hline & & Aggressive & 63 & 67 & 67 \\
\hline & \multirow[t]{4}{*}{ Self } & Anxious/Depressed & 52 & 50 & 50 \\
\hline & & Withdrawn/Depressed & 50 & 51 & 55 \\
\hline & & Rule-Breaking & 52 & 50 & 51 \\
\hline & & Aggressive & 62 & 50 & 59 \\
\hline \multirow[t]{12}{*}{2} & \multirow[t]{4}{*}{ Mother } & Anxious/Depressed & 66 & 62 & 53 \\
\hline & & Withdrawn/Depressed & $70 *$ & $73 *$ & 58 \\
\hline & & Rule-Breaking & 64 & 67 & 57 \\
\hline & & Aggressive & $70 *$ & $73 *$ & 61 \\
\hline & \multirow[t]{4}{*}{ Teacher } & Anxious/Depressed & $91 *$ & 55 & 64 \\
\hline & & Withdrawn/Depressed & $81 *$ & 69 & 60 \\
\hline & & Rule-Breaking & 69 & 63 & 50 \\
\hline & & Aggressive & $92 *$ & 63 & 60 \\
\hline & \multirow[t]{4}{*}{ Self } & Anxious/Depressed & $79 *$ & 67 & 53 \\
\hline & & Withdrawn/Depressed & $74 *$ & 63 & 58 \\
\hline & & Rule-Breaking & 68 & 64 & 57 \\
\hline & & Aggressive & $82 *$ & 60 & 61 \\
\hline \multirow[t]{12}{*}{3} & \multirow[t]{4}{*}{ Mother } & Anxious/Depressed & 65 & 60 & 60 \\
\hline & & Withdrawn/Depressed & $73 *$ & $70 *$ & $70 *$ \\
\hline & & Rule-Breaking & 68 & $70 *$ & $70 *$ \\
\hline & & Aggressive & 68 & 69 & 69 \\
\hline & \multirow[t]{4}{*}{ Teacher } & Anxious/Depressed & 51 & 50 & 59 \\
\hline & & Withdrawn/Depressed & 62 & 50 & $70 *$ \\
\hline & & Rule-Breaking & 63 & 62 & $71 *$ \\
\hline & & Aggressive & 53 & 62 & 69 \\
\hline & \multirow[t]{4}{*}{ Self } & Anxious/Depressed & 50 & 50 & 50 \\
\hline & & Withdrawn/Depressed & 50 & 50 & 50 \\
\hline & & Rule-Breaking & 50 & 50 & 50 \\
\hline & & Aggressive & 50 & 50 & 50 \\
\hline \multirow[t]{3}{*}{4} & \multirow[t]{3}{*}{ Mother } & Anxious/Depressed & 50 & 50 & 50 \\
\hline & & Withdrawn/Depressed & 66 & 51 & 50 \\
\hline & & Rule-Breaking & 68 & 57 & 62 \\
\hline
\end{tabular}




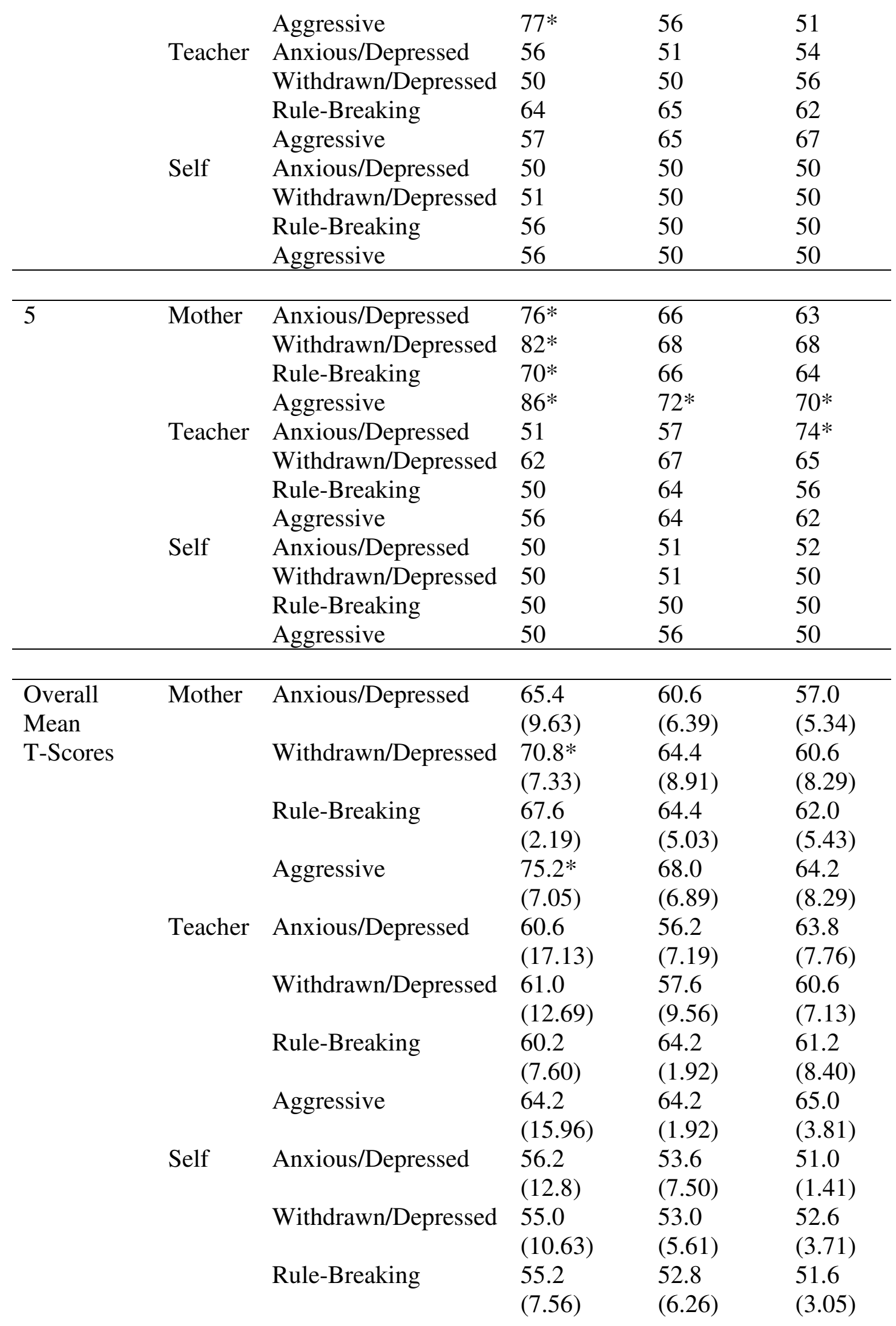


Aggressive

*Clinical range
60.0

(13.27)
54.6

(7.40)
54.0

(5.52) 
Table 5. Disruptive Behavior Disorder Rating Scale and Childhood Depression Inventory Scores

\begin{tabular}{|c|c|c|c|c|}
\hline Participant & Measure & $\begin{array}{c}\text { Pre- } \\
\text { Treatment }\end{array}$ & $\begin{array}{c}\text { Post- } \\
\text { Treatment }\end{array}$ & Follow-Up \\
\hline \multirow{6}{*}{1} & Mother CDI & $22 *$ & $30 *$ & 14 \\
\hline & Child CDI & 13 & 9 & 7 \\
\hline & Mother -ODD & $8^{*}$ & 1 & 6 \\
\hline & Child - ODD & 0 & 0 & 0 \\
\hline & Mother -CD & $5 *$ & 0 & 0 \\
\hline & Child - CD & 0 & 0 & 0 \\
\hline \multirow[t]{6}{*}{2} & Mother CDI & 16 & $24 *$ & 7 \\
\hline & Child CDI & 21 & $27 *$ & $27 *$ \\
\hline & Mother -ODD & $6^{*}$ & $4 *$ & 3 \\
\hline & Child - ODD & $5^{*}$ & 2 & 0 \\
\hline & Mother -CD & 1 & 1 & 0 \\
\hline & Child-CD & 0 & 0 & 0 \\
\hline \multirow[t]{6}{*}{3} & Mother CDI & 15 & 10 & 7 \\
\hline & Child CDI & 7 & 0 & 0 \\
\hline & Mother -ODD & $8^{*}$ & 1 & $6^{*}$ \\
\hline & Child- ODD & 2 & 1 & 0 \\
\hline & Mother -CD & $5^{*}$ & 0 & $3^{*}$ \\
\hline & Child-CD & 0 & 0 & 0 \\
\hline \multirow[t]{6}{*}{4} & Mother CDI & 12 & 7 & 5 \\
\hline & Child CDI & 3 & 1 & 0 \\
\hline & Mother -ODD & $4^{*}$ & 0 & 0 \\
\hline & Child- ODD & 0 & 0 & 0 \\
\hline & Mother -CD & 1 & 0 & 0 \\
\hline & Child-CD & 0 & 0 & 0 \\
\hline \multirow[t]{6}{*}{5} & Mother CDI & 21 & 15 & 19 \\
\hline & Child CDI & 7 & 9 & 6 \\
\hline & Mother -ODD & $7 *$ & 3 & $4 *$ \\
\hline & Child- ODD & 0 & 0 & 0 \\
\hline & Mother -CD & $3 *$ & 0 & $3 *$ \\
\hline & Child-CD & 0 & 0 & 0 \\
\hline \multirow[t]{7}{*}{ Overall Means } & Mother CDI & 17.2 & 17.2 & 12.4 \\
\hline & & $(4.21)$ & $(9.63)$ & $(6.47)$ \\
\hline & Child CDI & 10.2 & 9.2 & 8.0 \\
\hline & & (7.01) & (10.83) & (11.11) \\
\hline & Mother -ODD & 6.6 & 1.8 & 3.8 \\
\hline & & $(1.67)$ & $(1.64)$ & (2.49) \\
\hline & Child- ODD & 1.4 & 0.6 & 0.0 \\
\hline
\end{tabular}




\begin{tabular}{cccc} 
& $(2.19)$ & $(0.89)$ & $(0.00)$ \\
Mother -CD & 3.0 & 0.2 & 1.2 \\
& $(2.00)$ & $(0.45)$ & $(1.64)$ \\
Child-CD & 0 & 0 & 0 \\
\hline
\end{tabular}

*Clinical range 
Table 6. Family Environment and Emotion Regulation Ratings

\begin{tabular}{|c|c|c|c|c|}
\hline Participant & Measure & $\begin{array}{c}\text { Pre- } \\
\text { Treatment }\end{array}$ & $\begin{array}{c}\text { Post- } \\
\text { Treatment }\end{array}$ & Follow-Up \\
\hline \multirow[t]{6}{*}{1} & Lability/Negativity & 44 & 35 & 37 \\
\hline & Emotion & 22 & 26 & 24 \\
\hline & Regulation & & & \\
\hline & Conflict & $70 *$ & $65^{*}$ & 59 \\
\hline & Cohesion & 53 & 60 & 46 \\
\hline & Expressiveness & 60 & 66 & 66 \\
\hline \multirow[t]{6}{*}{2} & Lability/Negativity & 48 & 46 & 40 \\
\hline & Emotion & 20 & 22 & 18 \\
\hline & Regulation & & & \\
\hline & Conflict & 38 & 32 & 38 \\
\hline & Cohesion & 46 & 53 & 46 \\
\hline & Expressiveness & 54 & 66 & 60 \\
\hline \multirow[t]{6}{*}{3} & Lability/Negativity & 35 & 35 & 36 \\
\hline & Emotion & 20 & 23 & 25 \\
\hline & Regulation & & & \\
\hline & Conflict & 59 & $65^{*}$ & $65^{*}$ \\
\hline & Cohesion & 38 & 46 & 38 \\
\hline & Expressiveness & 66 & 66 & 66 \\
\hline \multirow[t]{6}{*}{4} & Lability/Negativity & 34 & 28 & 29 \\
\hline & Emotion & 22 & 22 & 24 \\
\hline & Regulation & & & \\
\hline & Conflict & 48 & 48 & 43 \\
\hline & Cohesion & $31 *$ & 46 & 46 \\
\hline & Expressiveness & 54 & 47 & 60 \\
\hline \multirow[t]{6}{*}{5} & Lability/Negativity & 38 & 36 & 36 \\
\hline & Emotion & 21 & 23 & 20 \\
\hline & Regulation & & & \\
\hline & Conflict & $75^{*}$ & $70 *$ & $70 *$ \\
\hline & Cohesion & 46 & 53 & 53 \\
\hline & Expressiveness & 47 & 60 & 54 \\
\hline \multirow{7}{*}{$\begin{array}{l}\text { Overall Means } \\
\quad \text { (SD) }\end{array}$} & Lability/Negativity & 39.8 & 36.0 & 35.6 \\
\hline & & $(6.02)$ & (6.44) & (4.04) \\
\hline & Emotion & 21.0 & 23.2 & 22.2 \\
\hline & Regulation & $(1.00)$ & $(1.64)$ & (3.03) \\
\hline & Conflict & 57.0 & 48.2 & 43.0 \\
\hline & & $(14.0)$ & $(16.75)$ & $(15.15)$ \\
\hline & Cohesion & 39.8 & 53.0 & 50.0 \\
\hline
\end{tabular}




\begin{tabular}{cccc} 
& $(9.63)$ & $(7.00)$ & $(10.58)$ \\
Expressiveness & 61.4 & 64.0 & 64.0 \\
& $(8.17)$ & $(10.27)$ & $(6.75)$ \\
\hline
\end{tabular}

*Clinical range 
Table 7. Ratings of Mother-Child Problem Solving Task

\begin{tabular}{|c|c|c|c|c|c|c|}
\hline Participant & Rater & $\begin{array}{c}\text { Pre- } \\
\text { Treatment }\end{array}$ & Week 5 & Week 8 & $\begin{array}{c}\text { Post- } \\
\text { Treatment }\end{array}$ & $\begin{array}{l}\text { Follow- } \\
\text { Up }\end{array}$ \\
\hline \multirow[t]{3}{*}{1} & Observer & 6 & 15 & 20 & 14 & 17 \\
\hline & Mother & 15 & 24 & 20 & 22 & 18 \\
\hline & Child & 15 & 26 & 18 & 27 & 21 \\
\hline \multirow[t]{3}{*}{2} & Observer & 18 & 9 & 17 & 17 & 14 \\
\hline & Mother & 20 & 23 & 19 & 27 & 24 \\
\hline & Child & 18 & 18 & 18 & 18 & 18 \\
\hline \multirow[t]{3}{*}{3} & Observer & 26 & 11 & 15 & 17 & 8 \\
\hline & Mother & 19 & 21 & 26 & 25 & 22 \\
\hline & Child & 20 & 9 & 28 & 26 & 24 \\
\hline \multirow[t]{3}{*}{4} & Observer & 5 & 9 & 14 & 6 & 6 \\
\hline & Mother & 17 & 26 & 23 & 26 & 20 \\
\hline & Child & 15 & 25 & 25 & 27 & 25 \\
\hline \multirow[t]{3}{*}{5} & Observer & 12 & 18 & 21 & 16 & 14 \\
\hline & Mother & 9 & 16 & 16 & 22 & 23 \\
\hline & Child & 15 & 14 & 14 & 26 & 28 \\
\hline \multirow{6}{*}{$\begin{array}{l}\text { Overall } \\
\text { Means } \\
(\mathrm{SD})\end{array}$} & Observer & 13.4 & 12.4 & 17.4 & 14.0 & 11.8 \\
\hline & & (8.76) & (3.97) & $(3.05)$ & (4.64) & $(4.60)$ \\
\hline & Mother & 16.0 & 22.0 & 20.8 & 24.4 & 21.4 \\
\hline & & (4.36) & (3.81) & (3.83) & (2.30) & $(2.41)$ \\
\hline & Child & 16.6 & 18.4 & 20.6 & 24.8 & 23.2 \\
\hline & & $(2.30)$ & (7.23) & (5.72) & $(3.83)$ & $(3.83)$ \\
\hline
\end{tabular}


Table 8. Mother and Child Ratings of Improvement

\begin{tabular}{|c|c|c|c|c|c|}
\hline Participant & Measure & Week 5 & Week 8 & $\begin{array}{c}\text { Post- } \\
\text { Treatment } \\
\end{array}$ & Follow-Up \\
\hline \multirow[t]{2}{*}{1} & $\begin{array}{l}\text { Mother Rating of } \\
\text { Improvement }\end{array}$ & 5 & 4 & 4 & 2 \\
\hline & $\begin{array}{l}\text { Child Rating of } \\
\text { Improvement }\end{array}$ & 4 & 3 & 5 & 4 \\
\hline \multirow[t]{2}{*}{2} & $\begin{array}{l}\text { Mother Rating of } \\
\text { Improvement }\end{array}$ & 4 & 4 & 5 & 5 \\
\hline & $\begin{array}{l}\text { Child Rating of } \\
\text { Improvement }\end{array}$ & 4 & 3 & 4 & 4 \\
\hline \multirow[t]{2}{*}{3} & $\begin{array}{l}\text { Mother Rating of } \\
\text { Improvement }\end{array}$ & 3 & 4 & 5 & 4 \\
\hline & $\begin{array}{l}\text { Child Rating of } \\
\text { Improvement }\end{array}$ & 3 & 5 & 5 & 5 \\
\hline \multirow[t]{2}{*}{4} & $\begin{array}{l}\text { Mother Rating of } \\
\text { Improvement }\end{array}$ & 4 & 4 & 5 & 5 \\
\hline & $\begin{array}{l}\text { Child Rating of } \\
\text { Improvement }\end{array}$ & 4 & 4 & 5 & 5 \\
\hline \multirow[t]{2}{*}{5} & $\begin{array}{l}\text { Mother Rating of } \\
\text { Improvement }\end{array}$ & 3 & 3 & 4 & 3 \\
\hline & $\begin{array}{l}\text { Child Rating of } \\
\text { Improvement }\end{array}$ & 4 & 4 & 5 & 4 \\
\hline \multirow[t]{2}{*}{ Overall } & $\begin{array}{l}\text { Mother Rating } \\
\text { of Improvement }\end{array}$ & 3.8 & 3.6 & 4.6 & 3.8 \\
\hline & $\begin{array}{l}\text { Child Rating of } \\
\text { Improvement }\end{array}$ & 3.8 & 4.2 & 4.8 & 4.4 \\
\hline \multirow{2}{*}{$\begin{array}{c}\text { Overall } \\
\text { Qualitative } \\
\text { Improvement }\end{array}$} & $\begin{array}{l}\text { Mother Rating } \\
\text { of Improvement }\end{array}$ & $\begin{array}{c}\text { Slightly } \\
\text { Improved }\end{array}$ & $\begin{array}{c}\text { Slightly } \\
\text { Improved }\end{array}$ & Improved & $\begin{array}{c}\text { Slightly } \\
\text { Improved }\end{array}$ \\
\hline & $\begin{array}{l}\text { Child Rating of } \\
\text { Improvement }\end{array}$ & $\begin{array}{l}\text { Slightly } \\
\text { Improved }\end{array}$ & $\begin{array}{c}\text { Slightly } \\
\text { Improved }\end{array}$ & Improved & $\begin{array}{l}\text { Slightly } \\
\text { Improved }\end{array}$ \\
\hline
\end{tabular}


Figure 1. Mother and Child Weekly Ratings of ODD Symptoms
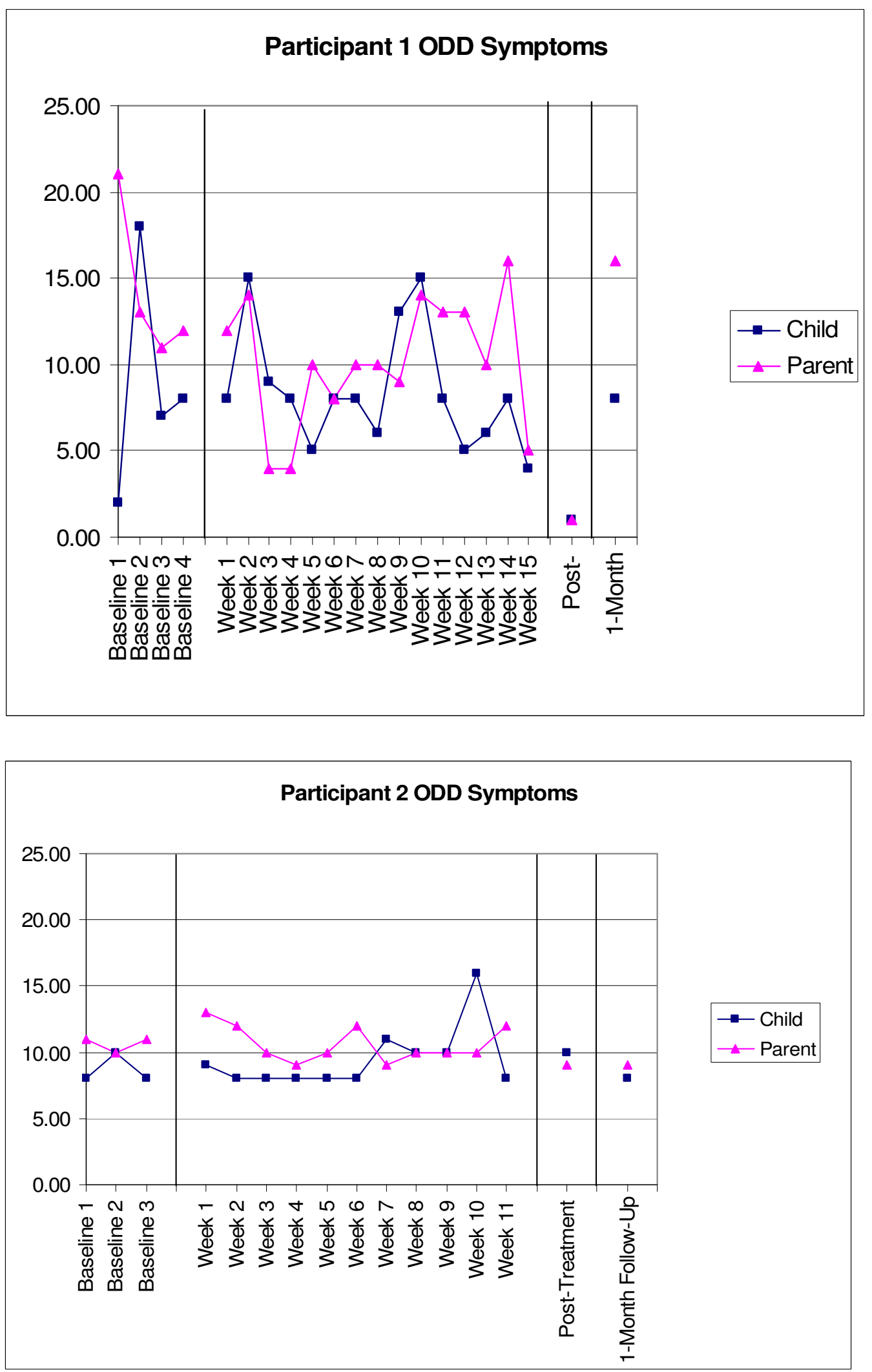

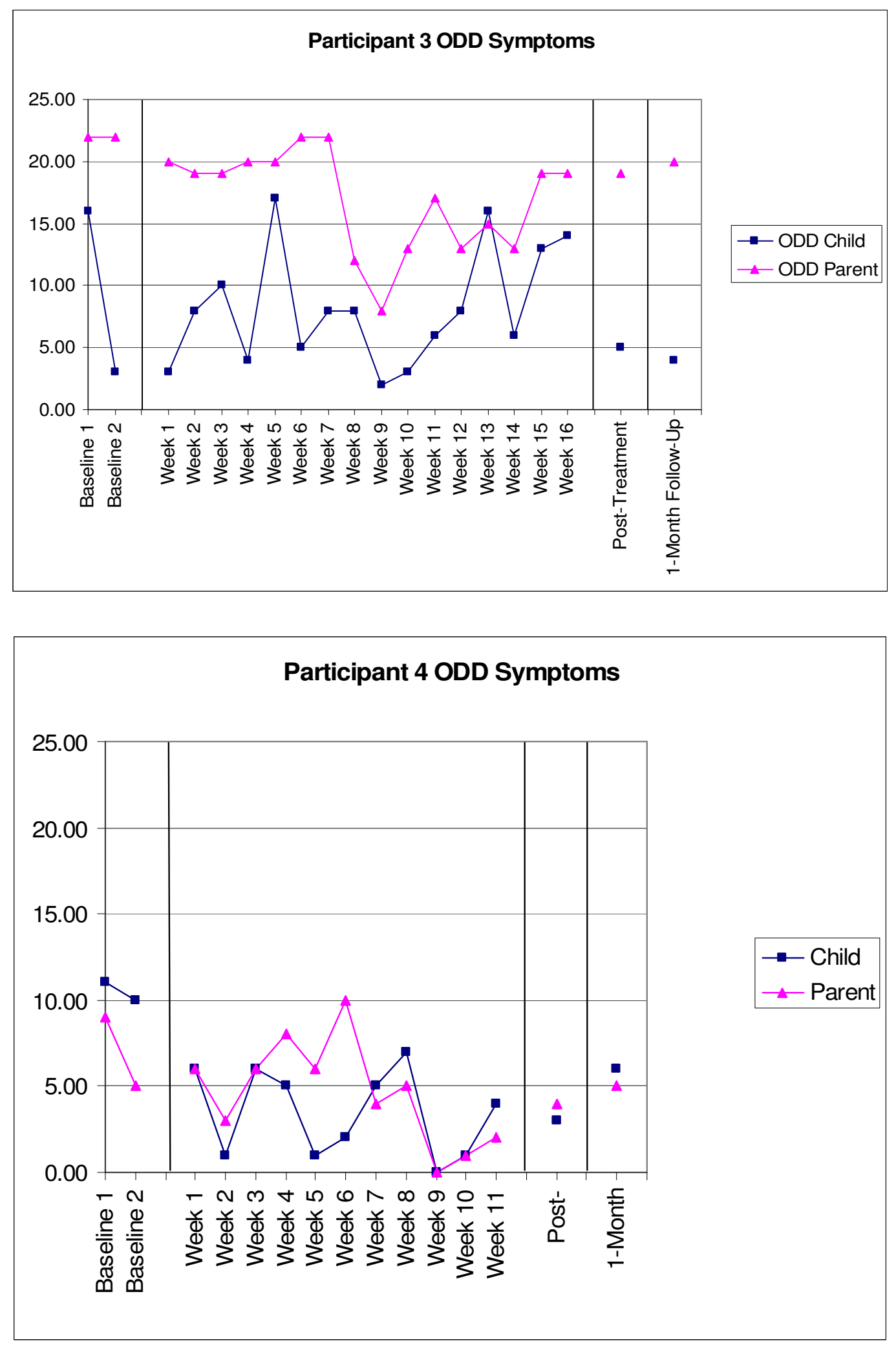


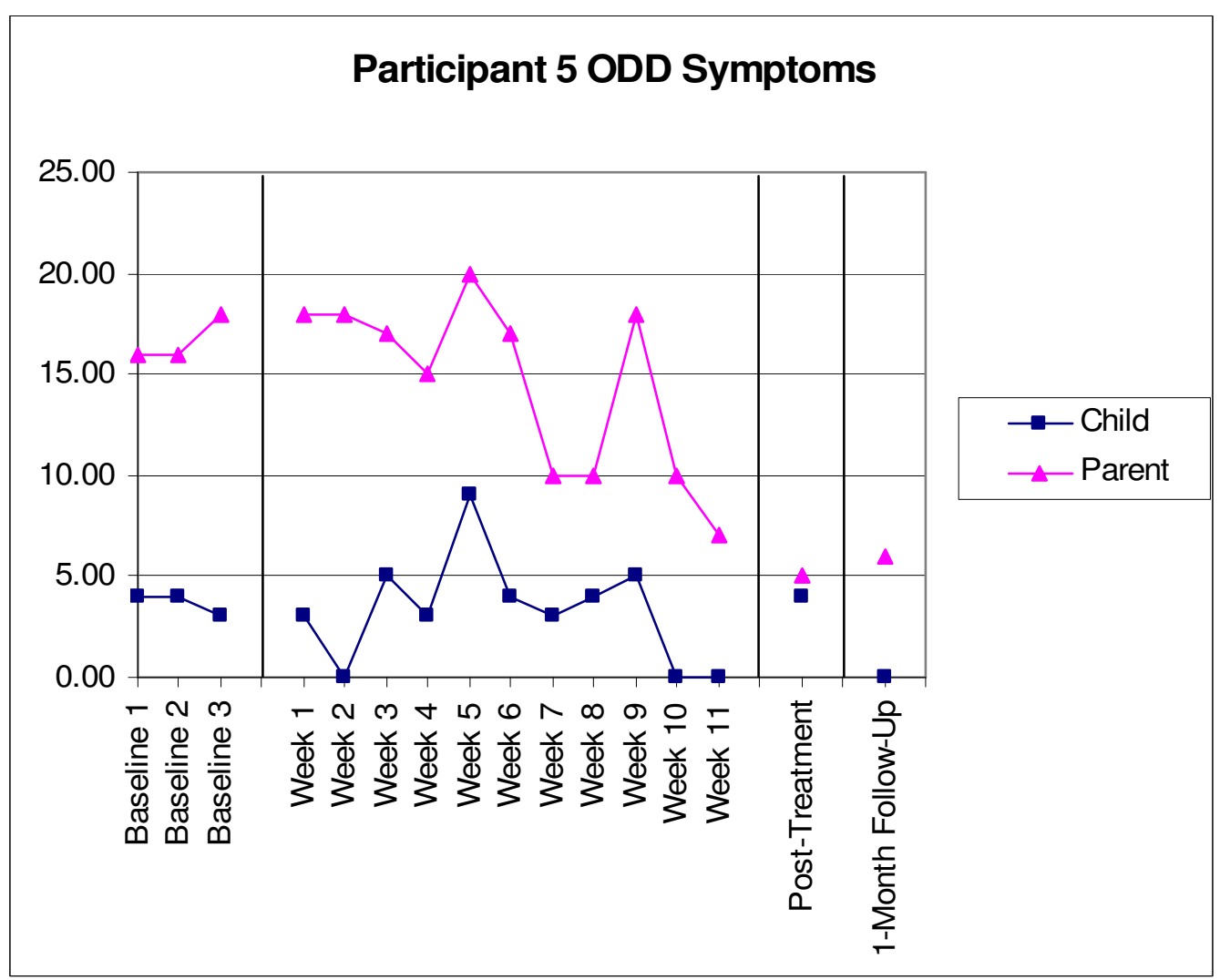


Figure 2. Mother and Child Weekly Ratings of Depression Symptoms
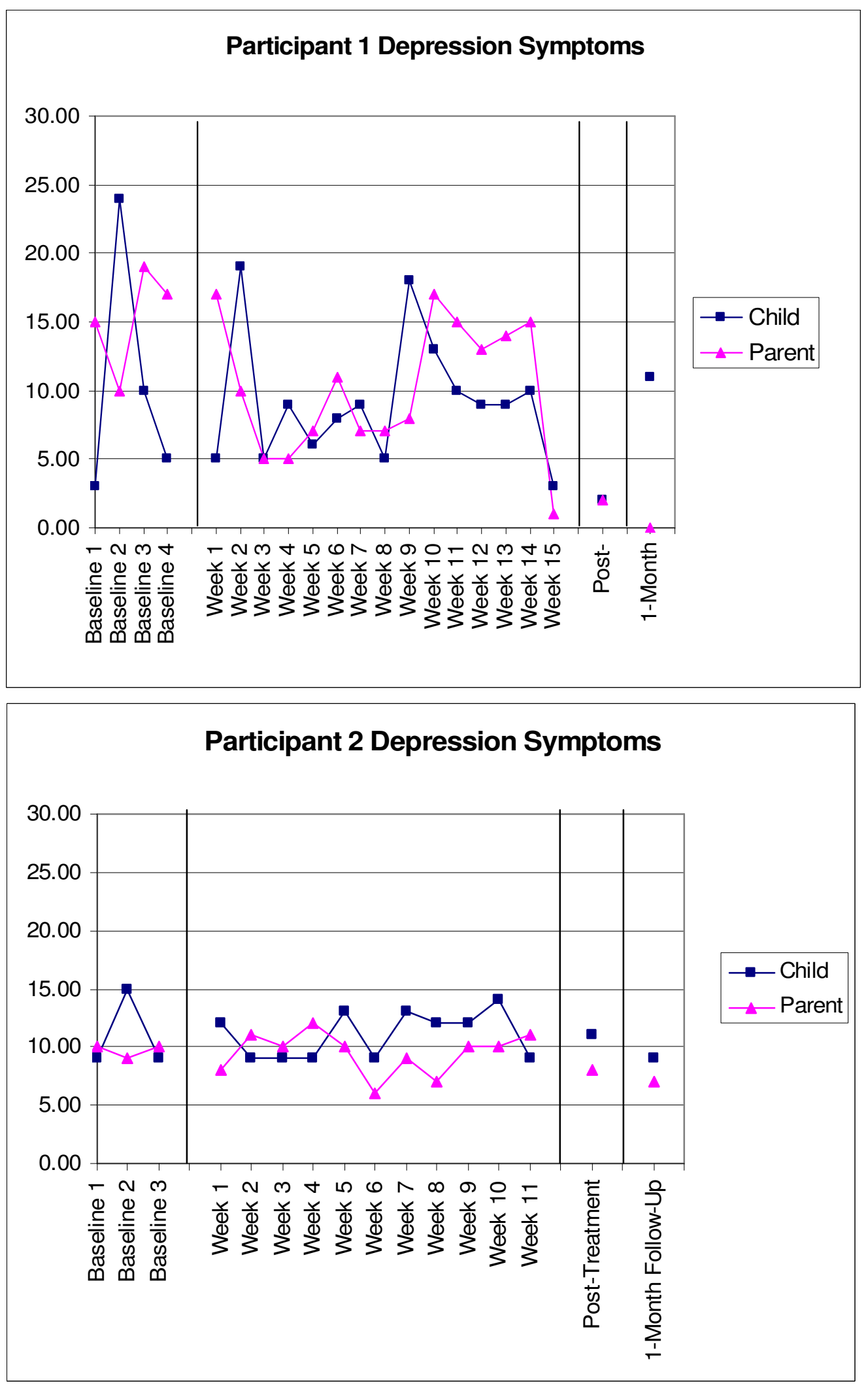

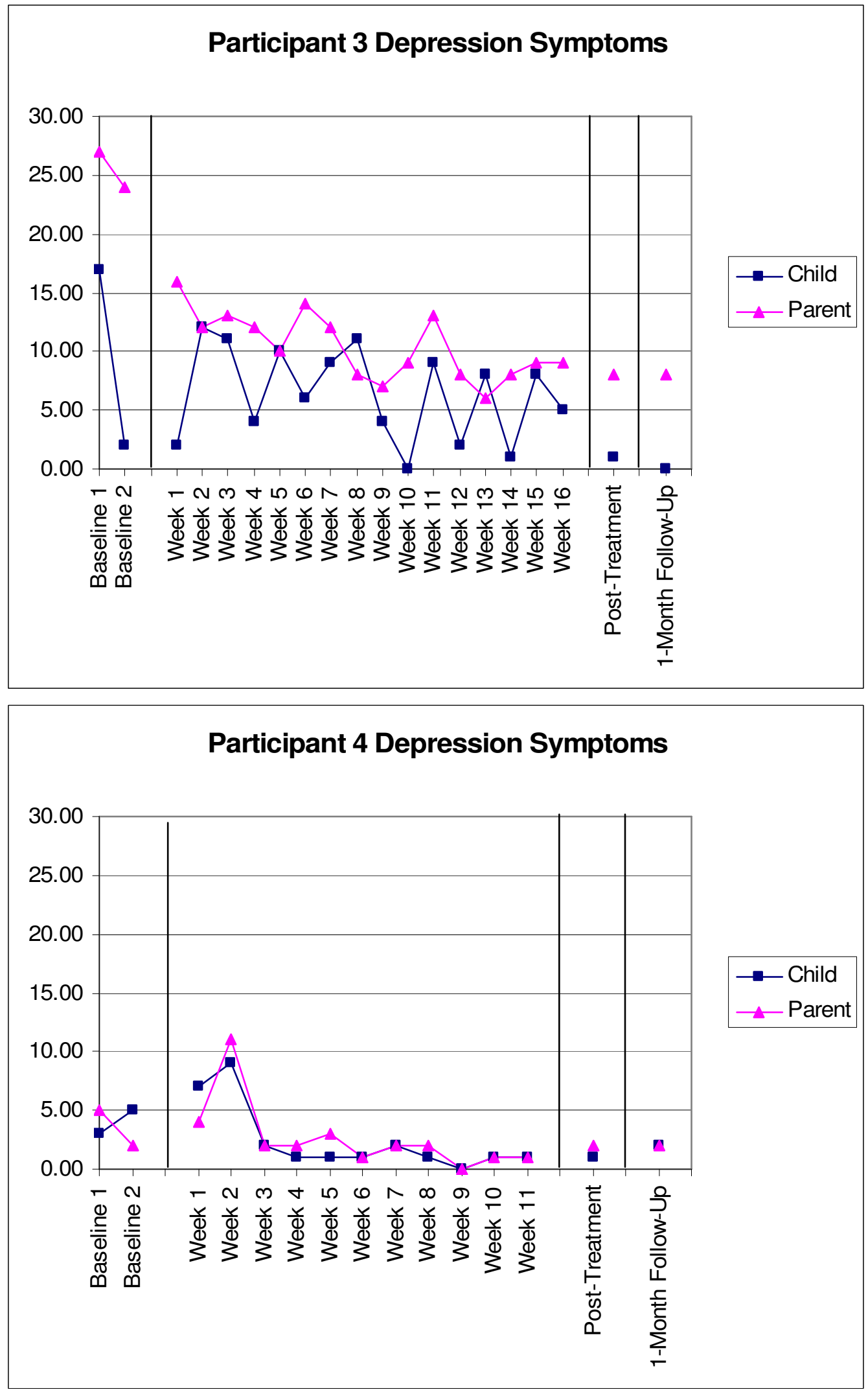


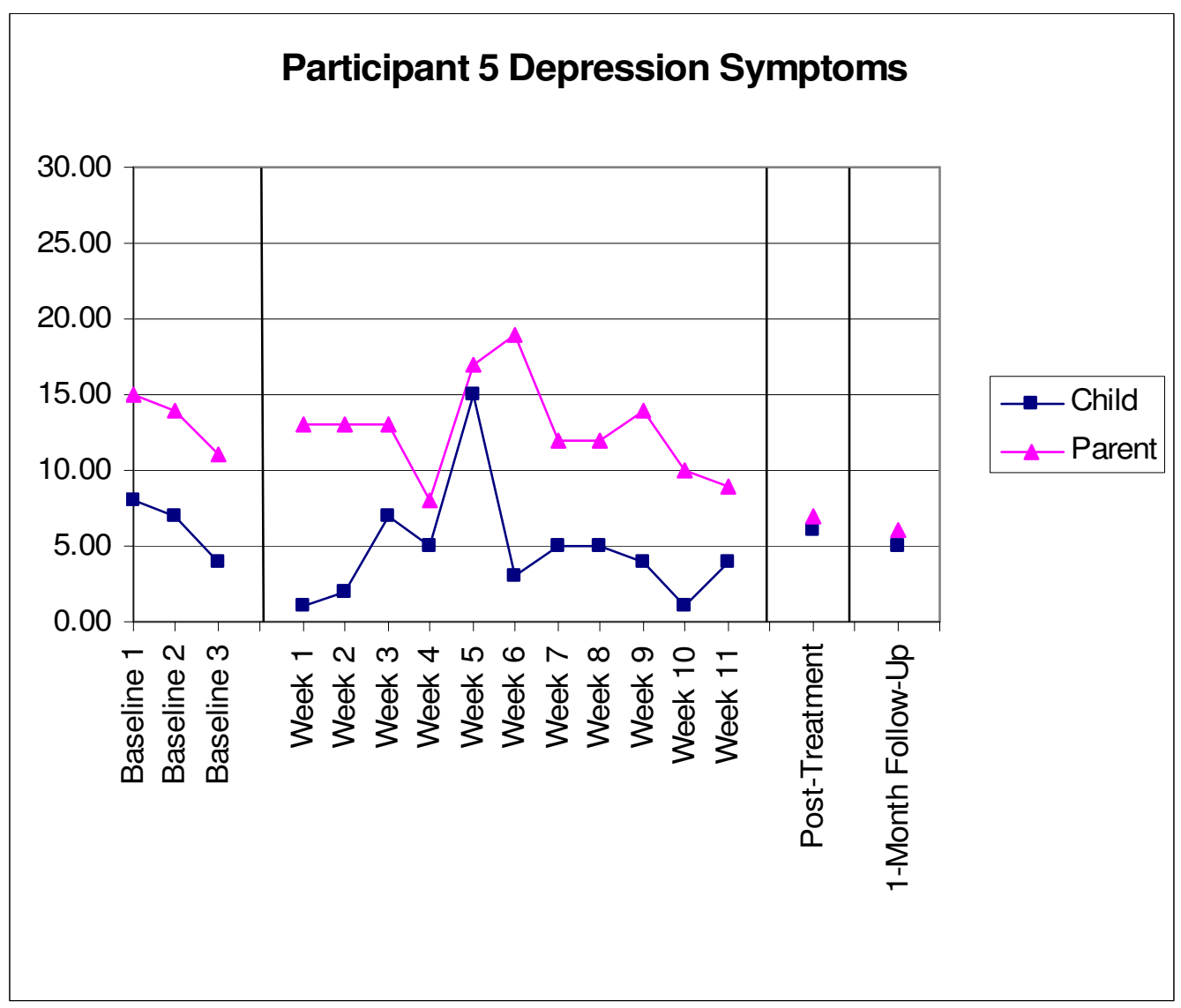


Figure 3. Clinician Ratings of Severity of Illness
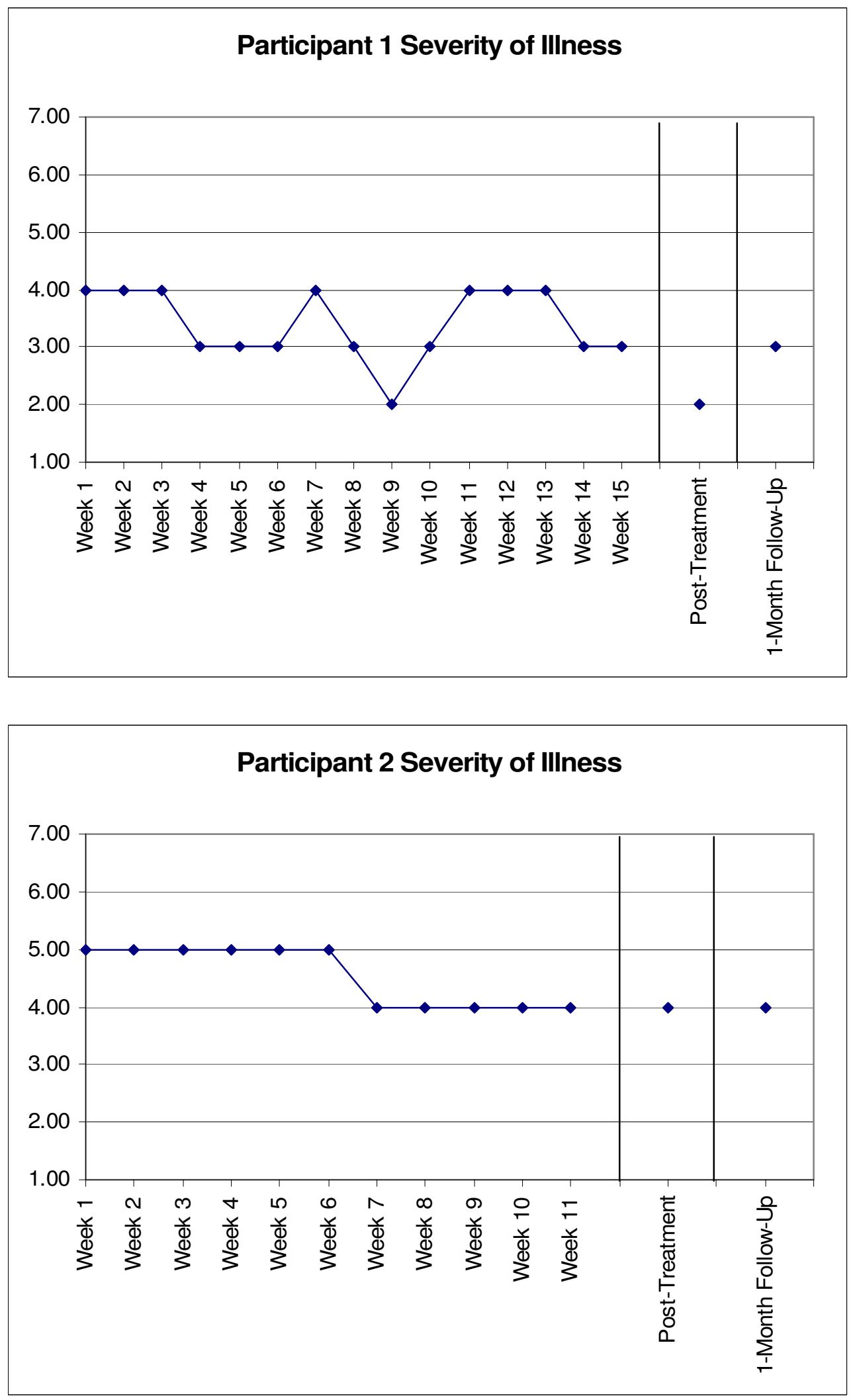

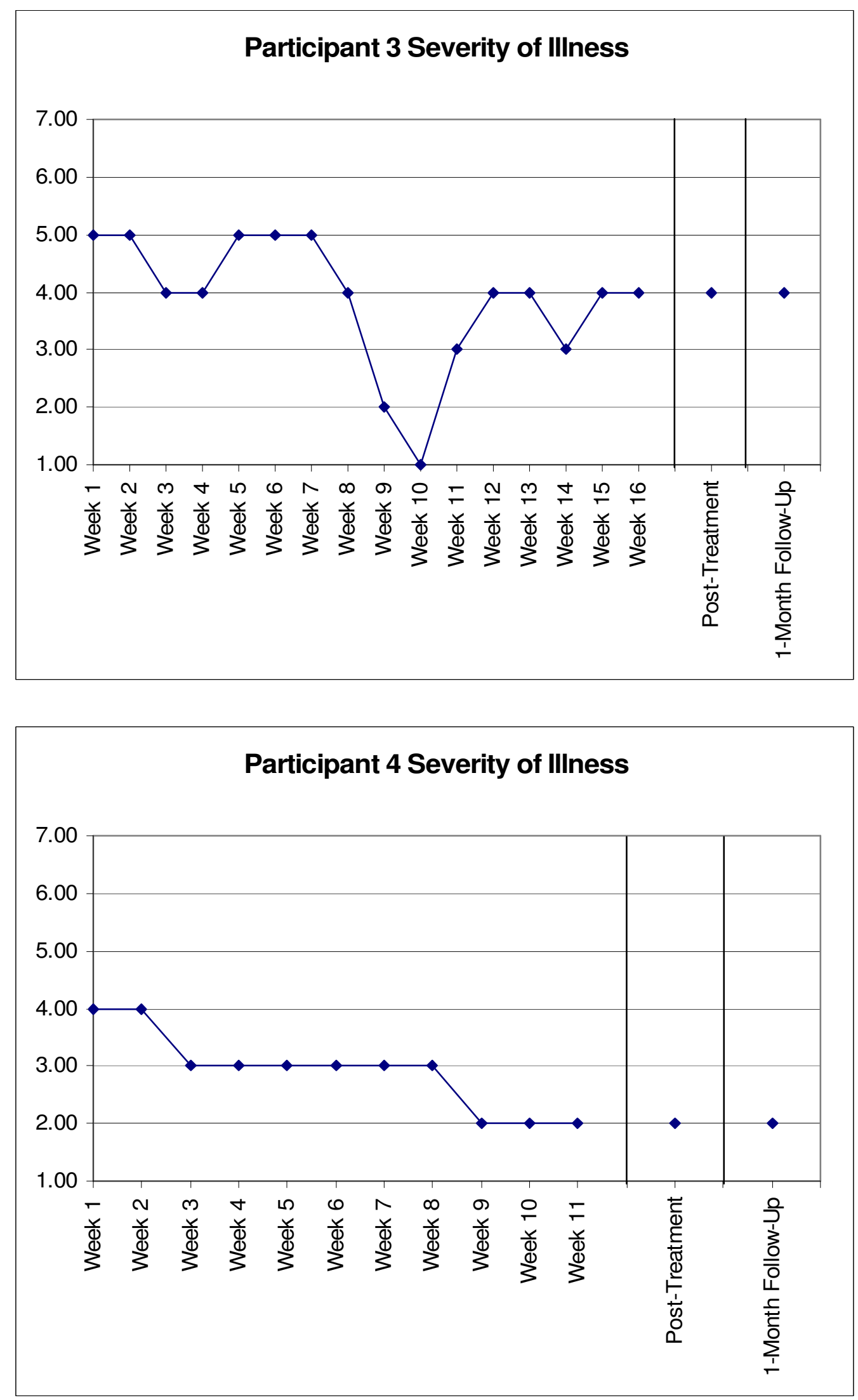


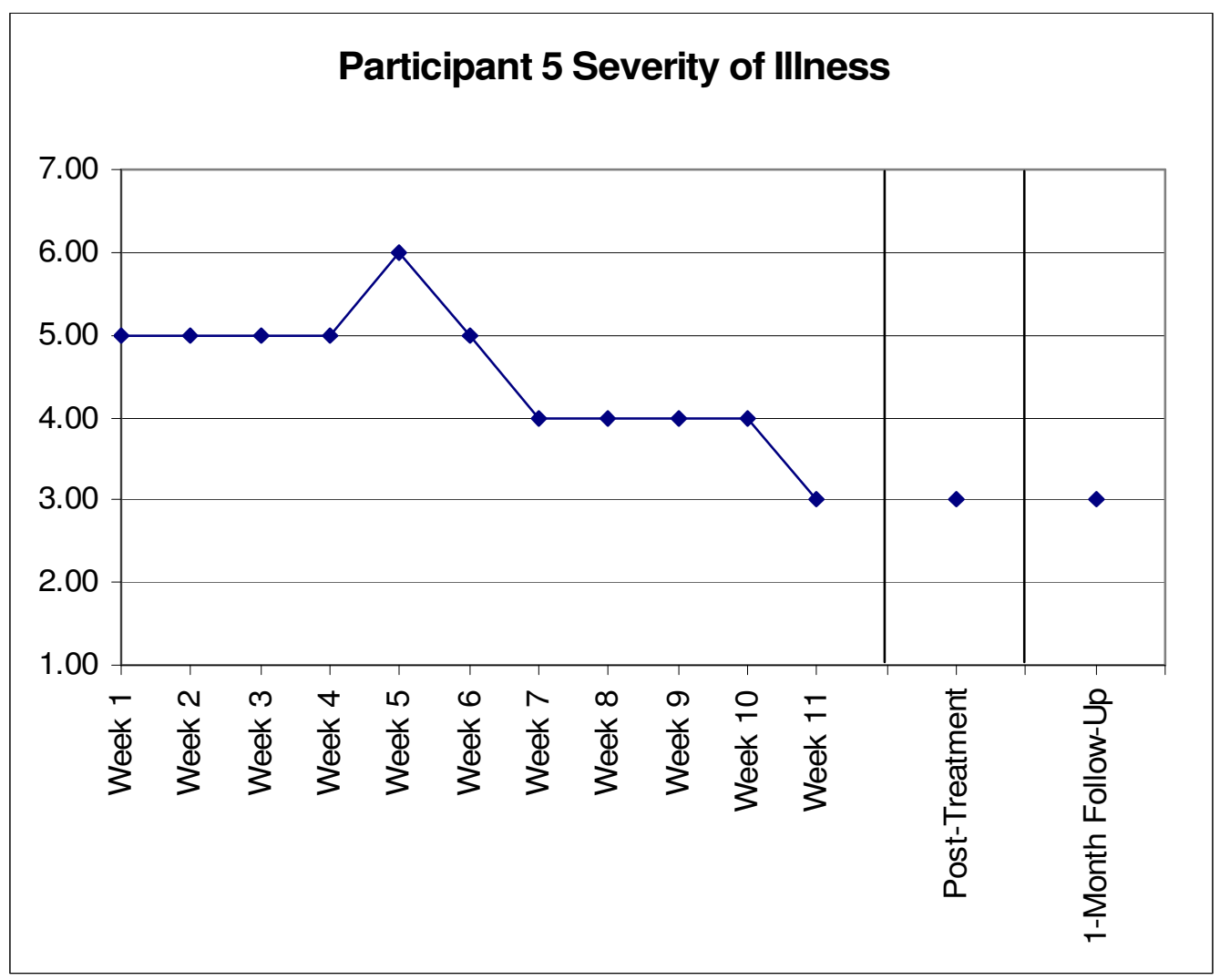


Figure 4. Clinician Ratings of Global Improvement
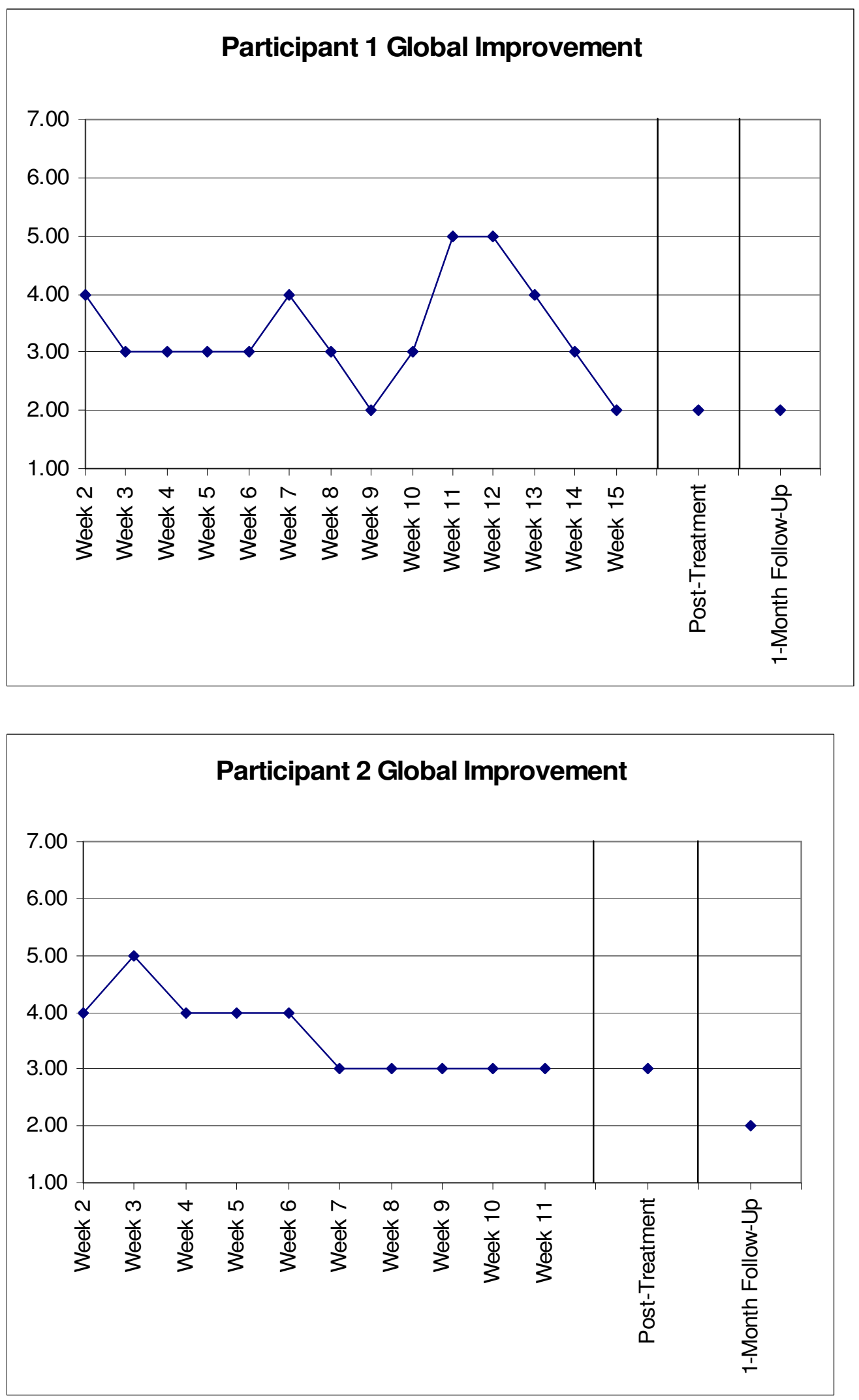

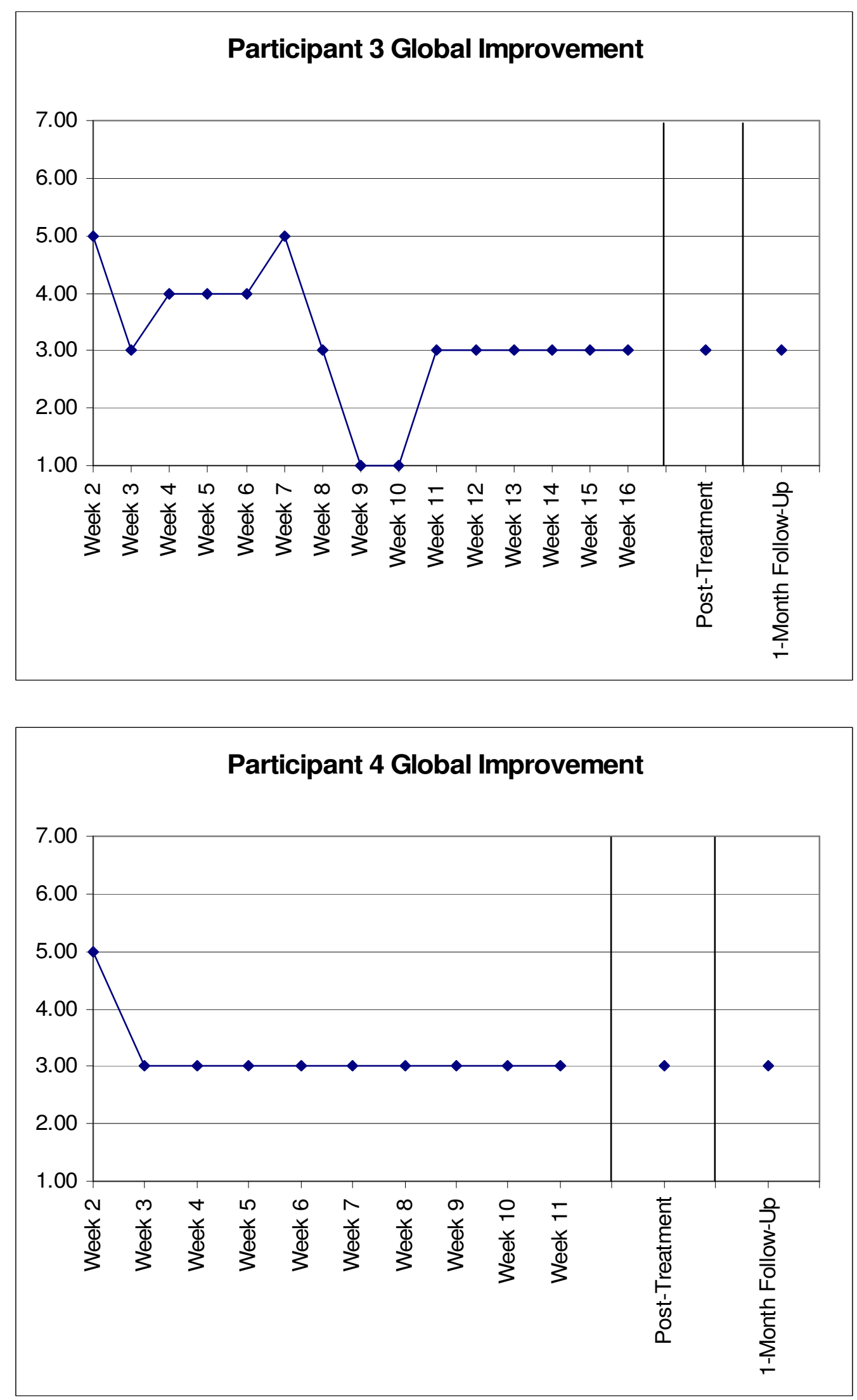


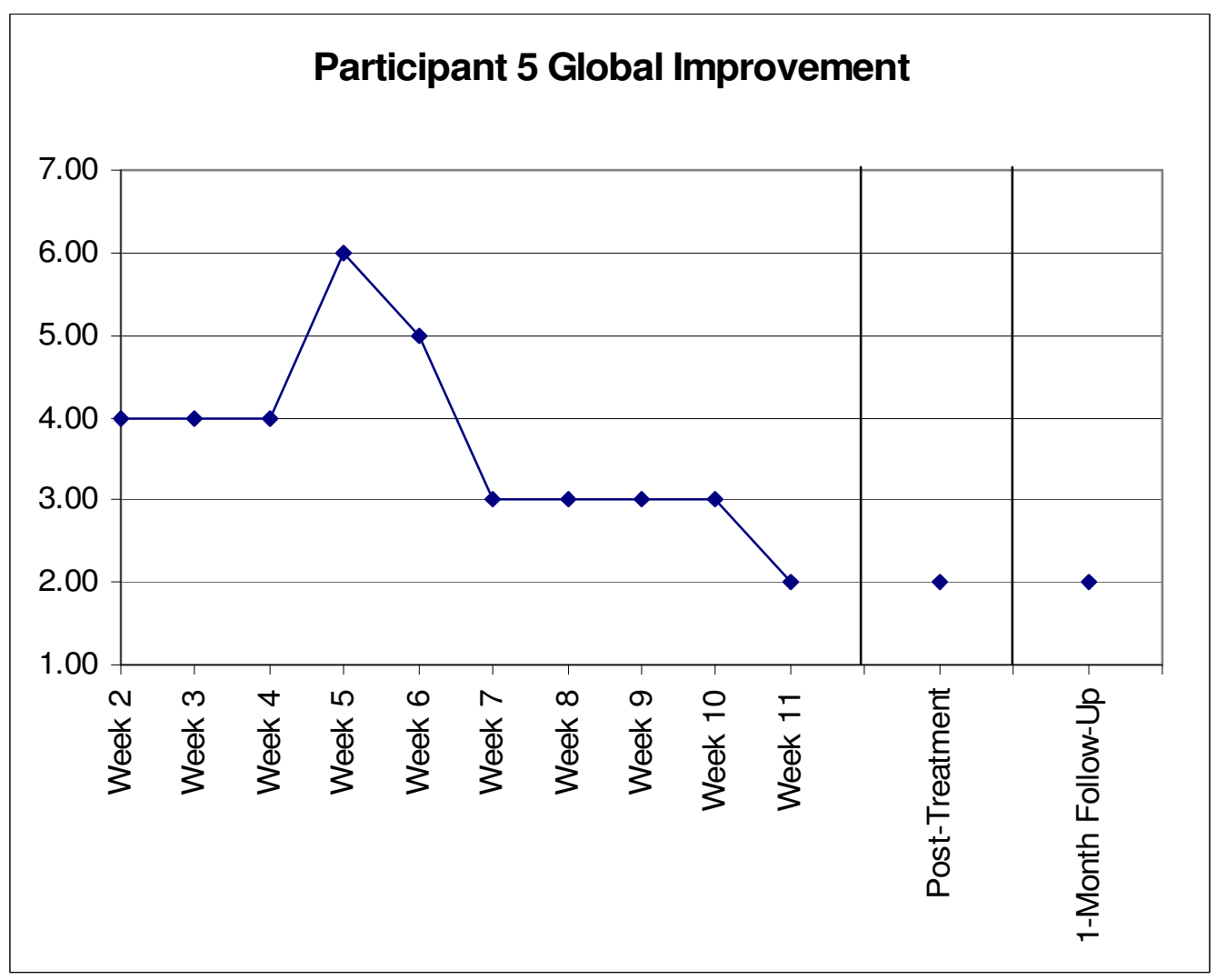


Appendix A 


\section{Virginia IIIIT) Tech}

VIRGINIA POLYTECHNIC INSTITUTE AND STATE UNIVERSITY

\author{
Department of Psychology \\ 460 Turner Street \\ Collegiate Square, Suite 207 \\ Blacksburg, Virginia 24060 \\ (540) 231-8276 Fax: (540) 231-8193
}

\section{Informed Consent for Participation in Research Projects Involving Human Subjects Informed Consent Form for Primary Caregiver}

\section{TITLE OF RESEARCH PROJECT: Treatment of Conduct Problems and Depression in Youth}

\section{PURPOSE OF THE RESARCH/PROJECT}

You are invited to participate in a study that will explore the effectiveness of treatments for children with conduct problems and mood problems. This project will compare your child's mood and behaviors from before treatment to their mood and behaviors after treatment, and again at a 1 month follow-up. The treatment involves eleven sessions (over a 12 week period) that will teach you and your child various skills for changing thoughts, feelings, and behaviors that are related to conduct problems and depression.

\section{PROCEDURES:}

To accomplish the goals of this project, you and your child will first be randomly (by chance) assigned to a waitlist that lasts between 2 and 4 weeks. It is necessary for us to have a brief waitlist condition so that we can determine if any change in your child's moods and behaviors occur simply due to the passage of time and the fact that you simply brought your child in to see us for an assessment. After the specified time on the wait list, treatment will begin. Most treatment sessions will be 1 hour long, but three sessions will be 1.5 hours long. After treatment, we will ask you and your child to return to the clinic for an assessment at the end of treatment and 1 month after treatment so that we can assess how things are going.

In order to determine whether the treatment is effective, you will be asked a variety of questions and administered a structured diagnostic interview about your child prior to the beginning of treatment, following treatment, and at 1-month follow-up. These questions will be focused on your child's level of depression and his or her everyday behavior (e.g., how well he or she gets along with others, how well he or she is doing in school, etc.). We will also ask you questions about your own moods and your family. Over the course of your participation you will occasionally ( 5 times) be asked to engage in a brief problem solving discussion with your child. All sessions you and your child attend at the clinic will be videotaped. Videotaping is done for the purpose of reliability, to document that interviewers and therapists carefully follow the research protocol.

We will also be asking you to give us permission to have your child's teacher complete a questionnaire regarding his/her behavior in school. This information will help us determine how your child's behavior at school changes as a result of treatment. In sum, the information collected from questionnaires, interviews, and teacher reports will help us to determine how much progress your child has made in treatment.

\section{RISKS:}

There may be some risks from your participation in this study. It is possible that you may become upset when asked to talk about thoughts, feelings, and behaviors that are related to your child's mood and 
conduct problems. However, to minimize this discomfort and to help you and your child manage the discomfort should it occur, all project staff are highly trained. The therapists and graduate student clinicians working on the project have experience working with children and families, and are being supervised by Dr. Ollendick, a licensed clinical psychologist. You do not have to answer any questions or discuss any topics that make you feel uneasy nor will you ever be asked to do anything you are not prepared to do. Of course, you may stop participating in the project at any time if you feel too uncomfortable or simply wish to discontinue the project.

\section{BENEFITS:}

Results of this study may help us determine whether this treatment is effective for youth with conduct problems and mood problems. Such a development would allow us to share this information with other mental health professionals and to assist them in working with other families. Although no guarantee of treatment outcome can be provided you, it is anticipated that this treatment will benefit both you and your child.

\section{EXTENT OF ANONYNITY AND CONFIDENTILAITY:}

Results of this study will be kept strictly confidential. At no time will we release your results to anyone without your written consent unless you have indicated that you will hurt or harm yourself or someone else, or that your child has indicated that someone is hurting or harming him/her, or that he/she has or intends to hurt himself/herself or someone else. In that situation, by law it would be necessary for us to report that information to the appropriate authorities. In all other cases, the information you provide will have your name removed and only a subject number will identify you during analyses and any write-up of the research.

The treatment sessions will be conducted by graduate clinicians enrolled in the doctoral program in clinical psychology at Virginia Tech. All clinicians will be supervised by Dr. Ollendick, a licensed clinical psychologist with over 35 years of experience working with such children and their families. As noted above, all sessions will be videotaped. The videotapes will be reviewed by research assistants (undergraduate and graduate students in the Psychology Department at Virginia Tech) and evaluated to ensure that the treatments are being implemented appropriately. The videotapes will be erased at the end of the study. The videotapes and all notes from the sessions will be kept in a locked file in the Child Study Center.

\section{COMPENSATION:}

Treatment will be offered to you free of charge. No direct compensation will be offered for your participation. However, if as a result of the project, it is determined that you should seek counseling, a list of local services will be provided

\section{FREEDOM TO WITHDRAW:}

You are free to withdraw from participation in this study at any time without penalty. Should you chose to withdraw, there will not be any penalty regardless of the reason for your decision to do so. You are also free to not answer any questions that you choose without penalty.

\section{USE OF RESEARCH DATA:}

The information from this research may be used for scientific or educational purposes. It may be presented at scientific meetings and/or published and reproduced in professional journals or books, or 
used for purposes that Virginia Tech's Department of Psychology considers proper in the interest of education, knowledge, or research. Only persons directly affiliated with the project, such as the Principal Investigator, Faculty Advisor, graduate students in psychology affiliated with the project, or trained undergraduate research assistants will have access to confidential participant information.

\section{APPROVAL OF RESEARCH:}

This research project has been approved by the Human Subjects Committee of the Department of Psychology and by the Institutional Review Board of Virginia Tech, as required for all university-based research projects.

\section{SUBJECT'S RESPONSIBILITIES:}

I voluntarily agree to participate in this study. I have the following responsibilities:

- Completing and returning the questionnaires

- $\quad$ Participation in three interviews about my child

- $\quad$ Participation in a problem solving task

- $\quad$ Attending therapy sessions

- Granting permission for my child to participate in the project

\section{SUBJECT'S PERMISSION:}

I have read and understand the above description of the study. I have had an opportunity to ask questions and have them answered. I hereby acknowledge the above and give my voluntary consent for my own and my child's participation in this study.

I further understand that if I participate I may withdraw at any time without penalty.

I understand that should I have any questions regarding this research and its conduct, I should contact any of the persons named below.

PRIMARY RESEARCHER: Jennifer C. Wolff, M.S. PHONE: 231-8511

FACULTY ADVISOR: Thomas H. Ollendick, Ph.D. PHONE: 231-6451

CHAIR, HSC: D.W. Harrison, Ph.D.

PHONE: 231-6581

CHAIR, IRB: David Moore, Ph.D.

PHONE: 231-4991

CHILD'S NAME:

PARENT/GUARDIAN'S NAME: Date:

PARENT/GUARDIAN'S SIGNATURE:

Date:

WITNESS: Date: 
Informed Consent for Participation in Research Projects Involving Human Subjects

Informed Consent Form for Teachers

TITLE OF RESEARCH PROJECT: Treatment of Conduct Problems and Depression in Youth

\section{PURPOSE OF THE RESARCH/PROJECT}

The major purpose of this study is to explore the effectiveness of treatments for children with conduct problems and mood problems. This project will compare your student's mood and behaviors from before treatment to their mood and behaviors after treatment, and again at a 1 month follow-up. The treatment involves eleven sessions (over a 12 week period) that will teach the child various skills for changing thoughts, feelings, and behaviors that are related to conduct problems and depression.

\section{PROCEDURES:}

Your involvement in this project will involve completing one questionnaire on three occasions that will take approximately 15 minutes to complete. This questionnaire will involve answering questions about the child's moods, behaviors, and so on. You will be asked to complete this questionnaire at your convenience and to return the form to the examiner in the enclosed, stamped, addressed envelope. This information will help us determine how the child's behavior at school changes as a result of treatment.

\section{RISKS:}

We anticipate minimal risks from your participation in this study. It is possible that you may become upset due to the nature of some of the questionnaire items. It should be clear however that these items apply to the child and not to you. You will not be asked to disclose anything about yourself and the parents have given us permission to request this information from you. Nonetheless, to minimize any risk you might experience, you will be provided with the telephone numbers for the Psychological Services Center and the Child Study Center of Virginia Tech should you wish to address this possible risk and/or seek additional advice. Of course, you may stop participating in the study at any time if you feel uncomfortable or simply wish to discontinue the project.

\section{BENEFITS:}

Results of this study may help us determine whether this treatment is effective for youth with conduct problems and mood problems. Such a development would allow us to share this information with other mental health professionals and to assist them in working with other families. The investigators will be available throughout the study and after completion of the study to address any questions or concerns that may arise for you, and to provide you with a summary of the research results. No promise or guarantee of benefits is being made to encourage you to participate.

\section{5. $\quad$ EXTENT OF ANONYNITY AND CONFIDENTILAITY:}

Results of this study will be kept strictly confidential. The researchers will not release your information to anyone without your written consent, and your responses will not be identified in publications or discussed in casual conversations. In all cases, the information you provide will have your name removed and only a subject number will identify you during analyses and any write-up of the research.

\section{COMPENSATION:}

No compensation will be offered for your participation. 


\section{FREEDOM TO WITHDRAW:}

You are free to withdraw from participation in this study at any time without penalty. Should you chose to withdraw, there will not be any penalty regardless of the reason for your decision to do so. You are also free to not answer any questions that you choose without penalty.

\section{USE OF RESEARCH DATA:}

The information from this research may be used for scientific or educational purposes. It may be presented at scientific meetings and/or published and reproduced in professional journals or books, or used for purposes that Virginia Tech's Department of Psychology considers proper in the interest of education, knowledge, or research. Only persons directly affiliated with the project, such as the Principal Investigator, Faculty Advisor, graduate students in psychology affiliated with the project, or trained undergraduate research assistants will have access to confidential participant information.

\section{9. $\quad$ APPROVAL OF RESEARCH:}

This research project has been approved by the Human Subjects Committee of the Department of Psychology and by the Institutional Review Board of Virginia Tech, as required for all university-based research projects.

10. SUBJECT'S RESPONSIBILITIES:

I voluntarily agree to participate in this study. I have the following responsibilities:

- Completing and returning the questionnaires

11. SUBJECT'S PERMISSION:

I have read and understand the above description of the study. I have had an opportunity to ask questions and have them answered. I hereby acknowledge the above and give my voluntary consent for my own and my child's participation in this study.

I further understand that if I participate I may withdraw at any time without penalty.

I understand that should I have any questions regarding this research and its conduct, I should contact any of the persons named below.

PRIMARY RESEARCHER: Jennifer C. Wolff, M.S. PHONE: 231-8511

FACULTY ADVISOR: Thomas H. Ollendick, Ph.D.PHONE: 231-6451

CHAIR, HSC: D.W. Harrison, Ph.D.

CHAIR, IRB: David Moore, Ph.D.

PHONE: 231-6581

PHONE: 231-4991

Date: 